\title{
EVOLUTION OF PLANE CURVES DRIVEN BY A NONLINEAR FUNCTION OF CURVATURE AND ANISOTROPY*
}

\author{
KAROL MIKULA ${ }^{\dagger}$ AND DANIEL ŠEVČOVIČ $\ddagger$
}

\begin{abstract}
In this paper we study evolution of plane curves satisfying a geometric equation $v=\beta(k, \nu)$, where $v$ is the normal velocity and $k$ and $\nu$ are the curvature and tangential angle of a plane curve $\Gamma$. We follow the direct approach and we analyze the so-called intrinsic heat equation governing the motion of plane curves obeying such a geometric equation. The intrinsic heat equation is modified to include an appropriate nontrivial tangential velocity functional $\alpha$. We show how the presence of a nontrivial tangential velocity can prevent numerical solutions from forming various instabilities. From an analytical point of view we present some new results on short time existence of a regular family of evolving curves in the degenerate case when $\beta(k, \nu)=\gamma(\nu) k^{m}, 0<m \leq 2$, and the governing system of equations includes a nontrivial tangential velocity functional.
\end{abstract}

Key words. nonlinear curve evolution, intrinsic heat equation, degenerate parabolic equations, tangential velocity, numerical solution

AMS subject classifications. 35K65, 65N40, 53C 80

\section{PII. S0036139999359288}

1. Introduction. The goal of this paper is to study curvature-driven evolution of a family of closed smooth plane curves. We consider the case when the normal velocity $v$ of an evolving family of plane curves $\Gamma^{t}: S^{1} \rightarrow \mathbb{R}^{2}, t \in(0, T)$, is a function of the curvature $k$ and the tangential angle $\nu$ :

$$
v=\beta(k, \nu) .
$$

In past years, geometric equations of the form (1.1) have attracted a lot of attention from both the theoretical and the practical point of view. There is a wide range of possible applications of geometric equations of the form (1.1). They arise from various applied problems in mathematical modeling and scientific computing, and they can be investigated in a purely mathematical context.

In the theory of phase interfaces separating solid and liquid phases, (1.1) corresponds to the so-called Gibbs-Thomson law governing the crystal growth in an undercooled liquid $[25,39,13]$. In the series of papers $[9,10,11]$ Angenent and Gurtin studied motion of phase interfaces. They proposed to study the equation of the form $\mu(\nu, v) v=h(\nu) k-g$, where $\mu$ is the kinetic coefficient and quantities $h, g$ arise from constitutive description of the phase boundary. The dependence of the normal velocity $v$ on the curvature $k$ is related to surface tension effects on the interface, whereas the dependence on $\nu$ (orientation of interface) introduces anisotropic effects into the model. In general, the kinetic coefficient $\mu$ may also depend on the velocity $v$ itself giving rise to a nonlinear dependence of the function $v=\beta(k, \nu)$ on $k$ and $\nu$. If the motion of an interface is very slow, then $\beta(k, \nu)$ is linear in $k$ (cf. [9]) and (1.1) corresponds to the classical mean curvature flow studied extensively from both

*Received by the editors July 16, 1999; accepted for publication (in revised form) June 14, 2000; published electronically January 19, 2001. This research was supported by grants $1 / 7132 / 20$ and 1/7677/20 from the Slovak Scientific Grant Agency VEGA.

http://www.siam.org/journals/siap/61-5/35928.html

${ }^{\dagger}$ Department of Mathematics, Slovak University of Technology, Radlinského 11, 81368 Bratislava, Slovak Republic (mikula@vox.svf.stuba.sk).

${ }^{\ddagger}$ Institute of Applied Mathematics, Faculty of Mathematics and Physics, Comenius University, 84248 Bratislava, Slovak Republic (sevcovic@fmph.uniba.sk). 
the mathematical (see, e.g., $[21,1,5,24]$ ) and the numerical point of view (see, e.g., $[18,16,31,35,36])$.

In the image processing the so-called morphological image and shape multiscale analysis is often used because of its contrast and affine invariance properties. It has been introduced by Sapiro and Tannenbaum [38] and Alvarez et al. [2, 3]. Analysis of image silhouettes (boundaries of distinguished shapes) leads to an equation of the form (1.1) without anisotropic part. Among various choices of a function $\beta(k)$ the so-called affine invariant scale space has special conceptual meaning and importance. In this case the velocity $v$ is given by $v=\beta(k)=k^{1 / 3}[2,38,12]$. In the context of image segmentation, various anisotropic models with $v=\beta(k, \nu)$ have been studied just recently $[27,30,15]$. For a comprehensive overview of applications of (1.1) in other applied problems, we refer to [42].

The analytical methods for mathematical treatment of (1.1) are strongly related to numerical techniques for computing curve evolutions. In the direct approach one seeks for a parameterization of the evolving family of curves. By solving the so-called intrinsic heat equation one can directly find a position vector of a curve (see, e.g., $[17,18,19,33,39,40])$. There are also other direct methods based on solution of a porous medium-like equation for curvature of a curve [31,32], a crystalline curvature approximation [22, 23, 44], special finite difference schemes [28, 29], and a method based on erosion of polygons in the affine invariant scale case [34]. By contrast to the direct approach, level set methods are based on introducing an auxiliary function whose zero level sets represent an evolving family of planar curves undergoing the geometric equation (1.1) (see, e.g., [36, 41, 42, 43, 26]). The other indirect method is based on the phase-field formulations (see, e.g., [14, 35, 20, 13]). The level set approach handles implicitly the curvature-driven motion, passing the problem to higher dimensional space. One can deal with splitting and/or merging of evolving curves in a robust way. However, from the computational point of view, level set methods are much more expensive than methods based on the direct approach.

In this paper we are concerned with the direct approach only. We consider the power-like function $\beta(k, \nu)=\gamma(\nu)|k|^{m-1} k$, where $\gamma(\nu)>0$ is a given anisotropy function and $m>0$. From the analytical perspective, the main purpose is to establish short time existence of a family of regular smooth plane curves satisfying the geometric equation (1.1). A short time existence result was obtained for the singular case in which $0<m<1$ as well as for the degenerate case in which $1<m \leq 2$. Let us emphasize that we needed additional geometric assumptions made on an initial curve in the degenerate case $1<m \leq 2$. Cases with higher powers of $m$ do not seem to be treatable by our techniques. On the other hand, recent results due to Andrews [4] show that the value $m=2$ is critical in the sense that, for higher powers of $m$, a solution need not necessarily be classical in points where the curvature vanishes.

In our approach, a family of evolving curves is represented by their position vector $x$ satisfying the geometric equation

$$
\partial_{t} x=\beta(k, \nu) \vec{N}+\alpha \vec{T} .
$$

Notice that the presence of an arbitrary tangential velocity functional $\alpha$ has no effect on the shape of evolving curves. The usual choice is therefore $\alpha=0$. From the numerical point of view, such a choice of $\alpha$ may lead to computational instabilities caused by merging of numerical grid points representing a discrete curve or by formation of the so-called swallow tails. In this paper we present an appropriate choice of a nontrivial 
tangential velocity $\alpha$. It turns out that if $\alpha$ is a solution of the nonlocal equation

$$
\frac{\partial \alpha}{\partial s}=k \beta(k, \nu)-\frac{1}{|\Gamma|} \int_{\Gamma} k \beta(k, \nu) d s,
$$

then material points are uniformly redistributed along the evolved curve. This choice of $\alpha$ results in a powerful numerical scheme having the property of uniform-in-time redistribution of grid points and preventing the computed numerical solution from forming the above-mentioned instabilities. Note that (1.2) can be transformed into a one-dimensional intrinsic heat equation (see (2.2)), and the functional $\alpha$ can be easily resolved from (1.3). In each time step we have to solve several linear tridiagonal systems in order to obtain a new position of the curve.

The outline of the paper is as follows. In section 2 we present the governing system of PDEs. Evolution of plane curves is parameterized by solutions of an intrinsic heat equation. We discuss the effect of a nontrivial tangential velocity on numerically computed solutions. Section 3 is focused on the analysis of the system of governing equations. The aim is to set up a closed system of parabolic equations solutions which include the curvature, the tangent angle, and the local length of a plane curve. The basic theory on short time existence of classical solutions is given in section 4. Here we consider only the case when $\beta_{k}^{\prime}$ is nondegenerate. We follow the abstract theory due to Angenent slightly modified for the case when a nontrivial tangential velocity functional is involved in the system of governing PDEs. Section 5 is devoted to the study of the singular case when $\beta(k, \nu)=\gamma(\nu)|k|^{m-1} k, m \neq 1$. We extend the result due to Angenent, Sapiro, and Tannenbaum obtained for the power $m=1 / 3$ to the general fast diffusion powers $0<m<1$ as well as for degenerate slow diffusion cases where $1<m \leq 2$. In section 6 we present a suitable choice of a tangential velocity leading to a powerful numerical scheme. We show how to construct a nontrivial tangential velocity as a nonlocal curve functional in such a way that relative local length (defined as the ratio of the local length to the total length of a curve) is constant along the evolution. A numerical scheme for full space-time discretization of the governing intrinsic heat equation is presented in section 7 . We derive this scheme by using the method of so-called flowing finite volumes. In section 8 we show several numerical solutions of the governing system of equations and we make a comparison between results obtained by considering the trivial and nontrivial tangential velocities, respectively. One can observe the importance of the presence of a suitable nontrivial tangential velocity functional in the governing system of equations for stability of numerical computations.

2. Preliminaries. Consider an embedded regular plane curve $\Gamma$ that can be parameterized by a $C^{2}$ smooth function $x: S^{1} \rightarrow \mathbb{R}^{2}$ such that $\Gamma=\operatorname{Image}(x)=$ $\{x(u), u \in[0,1]\}$ and $\left|\partial_{u} x\right|>0$. One can define the unit tangent vector $\vec{T}=\partial_{u} x /\left|\partial_{u} x\right|$ and the unit normal vector $\vec{N}$ in such a way that $\vec{T} \wedge \vec{N}=1$, where $\vec{a} \wedge \vec{b}$ is the determinant of the $2 \times 2$ matrix with column vectors $\vec{a}, \vec{b}$. Henceforth, we will denote $\vec{a} . \vec{b}$ as the Euclidean inner product of two vectors. By $|\vec{a}|=(\vec{a} \cdot \vec{a})^{1 / 2}$ we denote the Euclidean norm of a vector $\vec{a}$. The derivative of a function $f=f(\xi)$ with respect to $\xi$ will be denoted by $\partial_{\xi} f$. The arc-length parameterization will be denoted by $s$. Clearly, $d s=\left|\partial_{u} x\right| d u$. By $k$ we denote the signed curvature of the curve $\Gamma=\operatorname{Image}(x)$ defined as

$$
k=\partial_{s} x \wedge \partial_{s}^{2} x=\frac{\partial_{u} x \wedge \partial_{u}^{2} x}{\left|\partial_{u} x\right|^{3}}
$$


then Frenet's formulae read as follows: $\partial_{s} \vec{T}=k \vec{N}, \partial_{s} \vec{N}=-k \vec{T}$. The angle $\nu$ of the tangential vector is given by $\nu=\arg (\vec{T})$, i.e., $(\cos \nu, \sin \nu)=\partial_{s} x$. To describe the time evolution $\left\{\Gamma^{t}\right\}, t \in[0, T)$ of an initial curve $\Gamma=\Gamma^{0}=\operatorname{Image}\left(x^{0}\right)$, we adopt the notation $\Gamma^{t}=\{x(u, t), u \in[0,1]\}, \quad t \in[0, T)$, where $x \in C^{2}\left(Q_{T}, \mathbb{R}^{2}\right)$ and $Q_{T}=$ $S^{1} \times[0, T)$. We will frequently identify $Q_{T}$ with $[0,1] \times[0, T)$ and the space $C^{l}\left(Q_{T}, \mathbb{R}^{2}\right)$ with the space of $C^{l}$ differentiable functions defined on $[0,1]$ and satisfying periodic boundary conditions. The main idea in describing a family of evolving plane curves $\Gamma^{t}, t>0$, satisfying the geometric equation (1.1) is to parameterize $\Gamma^{t}$ by a solution $x \in C^{2}\left(Q_{T}, \mathbb{R}^{2}\right)$ of the so-called intrinsic heat equation

$$
\frac{\partial x}{\partial t}=\frac{1}{\theta_{1}} \frac{\partial}{\partial s}\left(\frac{1}{\theta_{2}} \frac{\partial x}{\partial s}\right), \quad x(., 0)=x^{0}(.),
$$

where $\theta_{1}, \theta_{2}$ are geometric quantities for the curve $\Gamma^{t}=\operatorname{Image}(x(., t))$, i.e., functions whose definition is independent of particular parameterization of $\Gamma^{t}$ and such that

$$
\theta_{1} \theta_{2}=\frac{k}{\beta(k, \nu)}
$$

By using (2.3) and Frenet's formulae, (2.2) can be rewritten in the following equivalent form:

$$
\frac{\partial x}{\partial t}=\beta \vec{N}+\alpha \vec{T}, \quad x(., 0)=x^{0}(.)
$$

where $\beta=\beta(k, \nu)$ is the normal velocity of the evolving curve and $\alpha$ is the tangential velocity given by

$$
\alpha=\frac{1}{\theta_{1}} \frac{\partial}{\partial s}\left(\frac{1}{\theta_{2}}\right) .
$$

The normal component $v$ of the velocity $\partial_{t} x$ is therefore equal to $\beta(k, \nu)$. By [12, Lemma 4.1] the family $\Gamma^{t}=\operatorname{Image}(x(., t))$ parameterized by a solution $x$ of the geometric equation (2.4) can be converted into a solution of $\partial_{t} x=\beta \vec{N}+\bar{\alpha} \vec{T}$ for any continuous function $\bar{\alpha}$ by changing the space parameterization of the original curve. In particular, it means that one can take $\bar{\alpha}=0$ without changing the shape of evolving curves. On the other hand, as can be observed from our numerical simulations, the presence of a suitable tangential velocity term $\alpha \vec{T}$ is necessary for construction of a numerical scheme capable of suitable redistribution of numerical grid points along a computed curve.

In [33] the authors studied the intrinsic heat equation (2.2) with $\theta_{1}=\theta_{2}=$ $(k / \beta(k))^{1 / 2}$. In this case, $(2.2)$ has the form $\partial_{t} x=\partial_{\bar{s}}^{2} x$, where $d \bar{s}=\theta_{1} d s$. Using this particular choice of $\theta_{1}, \theta_{2}$ we were able to simulate the evolution of plane convex and nonconvex curves for the case where $v=|k|^{m-1} k$. Satisfactory results were obtained only for $0<m \leq 1$, whereas various numerical instabilities appeared for the case $m>1$. The mathematical explanation for such a behavior is very simple. If $\theta_{1}=\theta_{2}=|k|^{\frac{m-1}{2}}$, then, by (2.5), $\alpha=\frac{m-1}{2}|k|^{m-3} k \partial_{s} k=\frac{1}{2} \partial_{s}\left(|k|^{m-1}\right)$. In the case $m>1$ numerical grid points were driven by the tangential velocity $\alpha \vec{T}$ toward pieces of the curve with the increasing curvature. It may lead to serious computational troubles. The effect of $\alpha$ is just the opposite when $0<m<1$.

Another possible choice of a nontrivial tangential velocity was studied by Deckelnick in [16] for the case $\beta(k)=k$. He proposed a governing PDE in the form 
$\partial_{t} x=\partial_{u}^{2} x /\left|\partial_{u} x\right|^{2}$. In this case $\alpha=-\partial_{u}\left(\left|\partial_{u} x\right|^{-1}\right)$, and thus $\theta_{2}=\theta_{1}^{-1}, \theta_{1}=\left|\partial_{u} x\right|$. This algorithm also has the property of a suitable redistribution of grid points along the computed curve. Notice that $\theta_{1}, \theta_{2}$ are not geometric quantities because of their dependence on a particular parameterization.

Note that the arc-length parameterization $s$ occurring in the intrinsic equation (2.2) depends on time $t$ and its initial position $u$ at $t=0$ via $d s=\left|\partial_{u} x\right| d u$. We can therefore rewrite (2.2) into the following Eulerian form:

$$
\frac{\partial x}{\partial t}=\frac{1}{\theta_{1}\left|\partial_{u} x\right|} \frac{\partial}{\partial u}\left(\frac{1}{\theta_{2}\left|\partial_{u} x\right|} \frac{\partial x}{\partial u}\right), \quad x(., 0)=x^{0}(.), \quad(u, t) \in Q_{T} .
$$

(2.6) seems to be a parabolic PDE for $x=x(u, t)$. However, as $\theta_{2}$ may depend on the curvature, the right-hand side of (2.6) may eventually contain the third-order derivative term $\partial_{u}^{3} x$. In the next section we will show how to overcome this difficulty by embedding (2.6) into a complete system of nonlinear parabolic equations.

3. Equations for geometric quantities. The goal of this section is to derive a system of PDEs governing the evolution of the curvature $k$ of $\Gamma^{t}=\operatorname{Image}(x(., t)), t \in$ $[0, T)$, and some other geometric quantities where the family of regular plane curves where $x=x(u, t)$ is a solution to the intrinsic heat equation (2.2). These equations will be used in order to derive a priori estimates of solutions. Notice that such an equation for the curvature is well known for the case when $\alpha=0$, and it reads as follows: $\partial_{t} k=\partial_{s}^{2} \beta+k^{2} \beta$, where $\beta=\beta(k, \nu)$ (cf. [21, 9]). Here we present a brief sketch of the derivation of the corresponding equations for the case of a nontrivial tangential velocity $\alpha$.

Let us denote $\vec{p}=\partial_{u} x$. Then, by using Frenet's formulae, one has

$$
\begin{aligned}
\partial_{t} \vec{p} & =\left|\partial_{u} x\right|\left(\left(\partial_{s} \beta+\alpha k\right) \vec{N}+\left(-\beta k+\partial_{s} \alpha\right) \vec{T}\right), \\
\vec{p} . \partial_{t} \vec{p} & =\left|\partial_{u} x\right| \vec{T} \cdot \partial_{t} \vec{p}=\left|\partial_{u} x\right|^{2}\left(-\beta k+\partial_{s} \alpha\right), \\
\vec{p} \wedge \partial_{t} \vec{p} & =\left|\partial_{u} x\right| \vec{T} \wedge \partial_{t} \vec{p}=\left|\partial_{u} x\right|^{2}\left(\partial_{s} \beta+\alpha k\right), \\
\partial_{t} \vec{p} \wedge \partial_{u} \vec{p} & =-\left|\partial_{u} x\right| \partial_{u}\left|\partial_{u} x\right|\left(\partial_{s} \beta+\alpha k\right)+\left|\partial_{u} x\right|^{3}\left(-\beta k+\partial_{s} \alpha\right),
\end{aligned}
$$

because $p_{u}=\partial_{u}^{2} x=\partial_{u}\left(\left|\partial_{u} x\right| \vec{T}\right)=\partial_{u}\left|\partial_{u} x\right| \vec{T}+k\left|\partial_{u} x\right|^{2} \vec{N}$. Since $\partial_{u}\left(\vec{p} \wedge \partial_{t} \vec{p}\right)=$ $\partial_{u} \vec{p} \wedge \partial_{t} \vec{p}+\vec{p} \wedge \partial_{u} \partial_{t} \vec{p}$, we have $\vec{p} \wedge \partial_{u} \partial_{t} \vec{p}=\partial_{u}\left(\vec{p} \wedge \partial_{t} \vec{p}\right)+\partial_{t} \vec{p} \wedge \partial_{u} \vec{p}$. As $k=\left(\vec{p} \wedge \partial_{u} \vec{p}\right)|\vec{p}|^{-3}$ (see (2.1)), we obtain

$$
\begin{aligned}
\partial_{t} k & =-3|p|^{-5}\left(\vec{p} . \partial_{t} \vec{p}\right)\left(\vec{p} \wedge \partial_{u} \vec{p}\right)+|\vec{p}|^{-3}\left(\left(\partial_{t} \vec{p} \wedge \partial_{u} \vec{p}\right)+\left(\vec{p} \wedge \partial_{u} \partial_{t} \vec{p}\right)\right) \\
& =-3 k|\vec{p}|^{-2}\left(\vec{p} . \partial_{t} \vec{p}\right)+2|\vec{p}|^{-3}\left(\partial_{t} \vec{p} \wedge \partial_{u} \vec{p}\right)+|\vec{p}|^{-3} \partial_{u}\left(\vec{p} \wedge \partial_{t} \vec{p}\right) .
\end{aligned}
$$

Finally, by applying identities (3.1), we end up with the second-order nonlinear parabolic PDE, the equation for the curvature:

$$
\partial_{t} k=\partial_{s}^{2} \beta+\alpha \partial_{s} k+k^{2} \beta, \quad k(., 0)=k^{0}(.) .
$$

Similarly, as in (2.6), the above equation can be rewritten into the Eulerian form

$$
\begin{gathered}
\frac{\partial k}{\partial t}=\frac{1}{\left|\partial_{u} x\right|} \frac{\partial}{\partial u}\left(\frac{1}{\left|\partial_{u} x\right|} \frac{\partial}{\partial u} \beta(k, \nu)\right)+\alpha \frac{1}{\left|\partial_{u} x\right|} \frac{\partial k}{\partial u}+k^{2} \beta(k, \nu), \\
k(., 0)=k^{0}(.),
\end{gathered}
$$


where $(u, t) \in Q_{T}$. The identities (3.1) can be used in order to derive an evolutionary equation for the local length $\left|\partial_{u} x\right|$. Indeed, $\left|\partial_{u} x\right|_{t}=\left(\partial_{u} x . \partial_{u} \partial_{t} x\right) /\left|\partial_{u} x\right|=$ $\left(\vec{p} . \partial_{t} \vec{p}\right) /\left|\partial_{u} x\right|$. By $(3.1)$ we have the local length equation

$$
\frac{\partial}{\partial t}\left|\partial_{u} x\right|=-\left|\partial_{u} x\right| k \beta+\frac{\partial \alpha}{\partial u}, \quad\left|\partial_{u} x(., 0)\right|=\left|\partial_{u} x^{0}(.)\right|,
$$

where $(u, t) \in Q_{T}$. In other words, $\partial_{t} d s=\left(-k \beta+\partial_{s} \alpha\right) d s$. By integrating (3.4) over the interval $[0,1]$ and taking into account that $\alpha$ satisfies periodic boundary conditions, we obtain the total length equation

$$
\frac{d}{d t} L^{t}+\int_{\Gamma^{t}} k \beta(k, \nu) d s=0
$$

where $L^{t}=L\left(\Gamma^{t}\right)$ is the total length of the curve $\Gamma^{t}, L^{t}=\int_{\Gamma^{t}} d s=\int_{0}^{1}\left|\partial_{u} x(u, t)\right| d u$. If $k \beta(k, \nu) \geq 0$, then the evolution of plane curves parameterized by a solution of $(2.2)$ represents a curve shortening flow, i.e., $L^{t_{2}} \leq L^{t_{1}} \leq L^{0}$ for any $0 \leq t_{1} \leq t_{2} \leq T$. The condition $k \beta(k, \nu) \geq 0$ is obviously satisfied in the case $\beta(k, \nu)=\gamma(\nu)|k|^{m-1} k$, where $m>0$ and $\gamma$ is a nonnegative anisotropy function.

The area enclosed by an embedded non-self-intersecting curve $\Gamma=\operatorname{Image}(x)$ can be computed as $A=\frac{1}{2} \int_{0}^{1} x \wedge \partial_{u} x d u$. Applying the identities (3.1) and taking into account that $0=\int_{0}^{1} \partial_{u}\left(x \wedge \partial_{t} x\right) d u=\int_{0}^{1}\left(\vec{p} \wedge \partial_{t} x+x \wedge \partial_{t} \vec{p}\right) d u$, where $\vec{p}=\partial_{u} x$, we obtain the area equation

$$
\frac{d}{d t} A^{t}+\int_{\Gamma^{t}} \beta(k, \nu) d s=0 .
$$

If $\beta(k, \nu)$ is nonnegative along the evolution, then the area is a nonincreasing function of the time.

Denote by $\left[\partial_{t}, \partial_{s}\right]$ the commutator of the differential operators $\partial_{t}$ and $\partial_{s}$, i.e., $\left[\partial_{t}, \partial_{s}\right]=\partial_{t} \partial_{s}-\partial_{s} \partial_{t}$. Since $d s=\left|\partial_{u} x\right| d u$ it follows from the local length equation (3.4) that the commutation relation

$$
\left[\partial_{t}, \partial_{s}\right]=\left(\beta k-\partial_{s} \alpha\right) \partial_{s} .
$$

Recall that the tangential vector $\nu$ to a curve $\Gamma=\operatorname{Image}(x)$ is given by $\nu=\arg (\vec{T})$, i.e., $(\cos \nu, \sin \nu)=\partial_{s} x$. From (3.7) we obtain $\partial_{t} \nu=\partial_{s} x \wedge \partial_{t} \partial_{s} x=\partial_{s} x \wedge \partial_{s} \partial_{t} x+$ $\left(\beta k-\partial_{s} \alpha\right)\left(\partial_{s} x \wedge \partial_{s} x\right)$. Applying Frenet's formulae and (2.4), we obtain the tangential vector equation

$$
\partial_{t} \nu=\partial_{s} \beta+\alpha k, \quad \nu(., 0)=\nu^{0}(.)
$$

Clearly,

$$
\partial_{s} \nu=\partial_{s} x \wedge \partial_{s}^{2} x=k
$$

Differentiating the curvature equation (3.2) with respect to $t$ and taking into account (3.8) yield an equation for the normal velocity $v=\beta(k, \nu)$, i.e., the normal velocity equation

$$
\begin{gathered}
\partial_{t} v=\beta_{k}^{\prime}\left(\partial_{s}^{2} v+\alpha \partial_{s} k+k^{2} v\right)+\beta_{\nu}^{\prime}\left(\partial_{s} v+\alpha k\right), \\
v(., 0)=v^{0}(.)=\beta\left(k^{0}(.), \nu^{0}(.)\right),
\end{gathered}
$$


where $\beta_{k}^{\prime}$ and $\beta_{\nu}^{\prime}$ are partial derivatives of the function $\beta=\beta(k, \nu)$ with respect to $k$ and $\nu$, respectively. Next we derive an equation for the gradient of the normal velocity $w=\partial_{s} v=\partial_{s} \beta(k, \nu)$. Using the commutation relation (3.7) we have

$$
\begin{aligned}
\partial_{t} w & =\partial_{t} \partial_{s} v=\partial_{s} \partial_{t} v+\left(v k-\partial_{s} \alpha\right) \partial_{s} v \\
& =\partial_{s}\left(\beta_{k}^{\prime}\left(\partial_{s} w+\alpha \partial_{s} k+k^{2} v\right)\right)+\beta_{\nu}^{\prime}(w+\alpha k)+\left(v k-\partial_{s} \alpha\right) w .
\end{aligned}
$$

Since

$$
w=\partial_{s} \beta(k, \nu)=\beta_{k}^{\prime} \partial_{s} k+\beta_{\nu}^{\prime} \partial_{s} \nu=\beta_{k}^{\prime} \partial_{s} k+\beta_{\nu}^{\prime} k,
$$

we end up with an equation for the gradient $w$ of the velocity $v$ :

$$
\begin{gathered}
\partial_{t} w=\partial_{s}\left(\beta_{k}^{\prime} \partial_{s} w\right)+\alpha \partial_{s} w+\partial_{s}\left(\beta_{k}^{\prime} k^{2} v+\beta_{\nu}^{\prime} w\right)+k v w \\
w(., 0)=w^{0}(.)=\partial_{s} v^{0}(.) .
\end{gathered}
$$

Now we are in a position to derive a closed system of governing equations for the geometric motion satisfying (1.2). It follows from (3.9) and (3.11) that $\partial_{t} \nu=$ $\beta_{k}^{\prime} \partial_{s}^{2} \nu+k\left(\alpha+\beta_{\nu}^{\prime}\right)$. Denoting $g=\left|\partial_{u} x\right|$, we can rewrite (3.3), (3.4), and (3.8) into the following closed form governing equations

$$
\begin{aligned}
& \frac{\partial k}{\partial t}=\frac{1}{g} \frac{\partial}{\partial u}\left(\frac{1}{g} \frac{\partial}{\partial u} \beta(k, \nu)\right)+\frac{\alpha}{g} \frac{\partial k}{\partial u}+k^{2} \beta(k, \nu), \\
& \frac{\partial \nu}{\partial t}=\frac{\beta_{k}^{\prime}(k, \nu)}{g} \frac{\partial}{\partial u}\left(\frac{1}{g} \frac{\partial \nu}{\partial u}\right)+k\left(\alpha+\beta_{\nu}^{\prime}(k, \nu)\right), \\
& \frac{\partial g}{\partial t}=-g k \beta(k, \nu)+\frac{\partial \alpha}{\partial u},
\end{aligned}
$$

$(u, t) \in[0,1] \times(0, T)$. A solution to (3.13) is subject to the initial conditions

$$
k(., 0)=k^{0}, \quad \nu(., 0)=\nu^{0}, \quad g(., 0)=g^{0}
$$

and periodic boundary conditions. Notice that the initial conditions for $k^{0}, \nu^{0}, g^{0}$ are related through the identity

$$
\partial_{u} \nu^{0}=g^{0} k^{0} .
$$

In general, the function $\alpha=\alpha(k, \nu, g)$ is a nonlinear function that will be determined later. In section 6 of this paper we present the choice of $\alpha$ leading to a powerful numerical algorithm preserving relative local length between numerical grid points.

4. Short time existence of solutions in the nondegenerate case. In this section we prove short time existence of a classical solution of the governing system of equations (3.13) by using the abstract result due to Angenent (cf. [8]).

Denote $\Phi=(k, \nu, g)^{T}$. Then (3.13) can be rewritten as a fully nonlinear PDE of the form

$$
\partial_{t} \Phi=f(\Phi), \quad \Phi(0)=\Phi^{0},
$$

where $f(\Phi)=F(\Phi, \alpha(\Phi))$ and $F(\Phi, \alpha)$ is the right-hand side of (3.13). Suppose that $\beta=\beta(k, \nu)$ is a $C^{2}$ smooth function such that

$$
0<\lambda_{-} \leq \beta_{k}^{\prime}(k, \nu) \leq \lambda_{+}<\infty \text { for any } k, \nu,
$$


where $\lambda_{ \pm}>0$ are constants and $\beta_{k}^{\prime}$ is a partial derivative of $\beta$ with respect to $k$. Given $0<\sigma<1$, we denote by $E_{0}, E_{1 / 2}, E_{1}$ the following Banach spaces:

$$
\begin{aligned}
E_{0} & =c^{\sigma}\left(S^{1}\right) \times c^{\sigma}\left(S^{1}\right) \times c^{1+\sigma}\left(S^{1}\right), \\
E_{1 / 2} & =c^{1+\sigma}\left(S^{1}\right) \times c^{1+\sigma}\left(S^{1}\right) \times c^{1+\sigma}\left(S^{1}\right), \\
E_{1} & =c^{2+\sigma}\left(S^{1}\right) \times c^{2+\sigma}\left(S^{1}\right) \times c^{1+\sigma}\left(S^{1}\right),
\end{aligned}
$$

where $c^{k+\sigma}, k=0,1,2$, is the little Hölder space, i.e., the closure of $C^{\infty}\left(S^{1}\right)$ in the topology of the Hölder space $C^{k+\sigma}\left(S^{1}\right)$ (see [6]). Let $\mathcal{O}_{i} \subset E_{i}$ be an open subset in $E_{i}$ such that $g>0$ for any $(k, \nu, g)^{T} \in \mathcal{O}_{i}, i=\frac{1}{2}, 1$. If we assume

$$
\alpha \in C^{1}\left(\mathcal{O}_{1 / 2}, c^{2+\sigma}\left(S^{1}\right)\right),
$$

then the mapping $f$ is a smooth mapping from $\mathcal{O}_{1} \subset E_{1}$ into $E_{0}$.

If the Fréchet derivative $d f(\bar{\Phi}) \in \mathcal{L}\left(E_{1}, E_{0}\right)$ belongs to the maximal regularity class $\mathcal{M}_{1}\left(E_{0}, E_{1}\right)$ for any $\bar{\Phi} \in \mathcal{O}_{1}$, then by [8, Theorem 2.7], (4.1) has a unique solution $\Phi \in Y^{T}=C\left([0, T], E_{1}\right) \cap C^{1}\left([0, T], E_{0}\right)$ on some small enough interval $[0, T]$. Recall that the class $\mathcal{M}_{1}\left(E_{0}, E_{1}\right) \subset \mathcal{L}\left(E_{1}, E_{0}\right)$ consists of those generators of analytic semigroups $A: D(A)=E_{1} \subset E_{0} \rightarrow E_{0}$ for which the linear equation $\partial_{t} \Phi=A \Phi+h(t)$, $0<t \leq 1, \Phi(0)=\Phi^{0}$, has a unique solution $\Phi \in Y^{1}$ for any $h \in C\left([0,1], E_{0}\right)$ and $\Phi^{0} \in E_{1}$. In other words, $\left(E_{0}, E_{1}\right)$ is a maximal parabolic regularity pair.

Theorem 4.1. Assume that $\left(k^{0}, \nu^{0}, g^{0}\right)^{T} \in \mathcal{O}_{1} \subset E_{1}$, where $k^{0}$ is the curvature, $\nu^{0}$ is the tangential vector, and $g^{0}=\left|\partial_{u} x^{0}\right|>0$ is the local length element of the initial regular curve $\Gamma^{0}=\operatorname{Image}\left(x^{0}\right)$. If $\beta=\beta(k, \nu)$ is a $C^{3}$ smooth function satisfying (4.2) and $\alpha$ obeys (4.4), then there exists a unique classical solution $\Phi=(k, \nu, g)^{T} \in C\left([0, T], E_{1}\right) \cap C^{1}\left([0, T], E_{0}\right)$ of the governing system of equations (3.13) defined on some small time interval $[0, T]$. Moreover, if $\Phi$ is a maximal solution defined on $\left[0, T_{\max }\right)$ and $T_{\max }<\infty$, then $\max |k(., t)| \rightarrow \infty$ as $t \rightarrow T_{\max }$.

Proof. Let $\Phi^{0} \in \mathcal{O}_{1}$ where $\mathcal{O}_{1} \subset E_{1}$ is an open and bounded subset of $E_{1}, g>0$, for any $(k, \nu, g)^{T} \in \mathcal{O}_{1}$. The linearization of $f$ at $\bar{\Phi}=(\bar{k}, \bar{\nu}, \bar{g})^{T} \in \mathcal{O}_{1}$ has the form $d f(\bar{\Phi})=d_{\Phi} F(\bar{\Phi}, \bar{\alpha})+d_{\alpha} F(\bar{\Phi}, \bar{\alpha}) d_{\Phi} \alpha(\bar{\Phi})$, where $\bar{\alpha}=\alpha(\bar{\Phi})$ and

$$
\begin{aligned}
d_{\Phi} F(\bar{\Phi}, \bar{\alpha}) \Phi & =\partial_{u}\left(\bar{D} \partial_{u} \Phi\right)+\bar{B} \partial_{u} \Phi+\bar{C} \Phi, \\
d_{\alpha} F(\bar{\Phi}, \bar{\alpha}) \alpha & =\left(\alpha \bar{g}^{-1} \partial_{u} \bar{k}, \alpha \bar{k}, \partial_{u} \alpha\right)^{T} ;
\end{aligned}
$$

$\bar{D}=\operatorname{diag}\left(\bar{D}_{11}, \bar{D}_{22}, 0\right), \bar{D}_{11}=\bar{D}_{22}=\beta_{k}^{\prime}(\bar{k}, \bar{\nu}) \bar{g}^{-2} \in C^{1+\sigma}\left(S^{1}\right)$, and $\bar{B}, \bar{C}$ are $3 \times 3$ matrices with $C^{\sigma}\left(S^{1}\right)$ smooth coefficients, $\bar{B}_{3 j}=0, \bar{C}_{3 j} \in C^{1+\sigma}$. By (3.9) we have $g^{-1} \partial_{u} \beta(k, \nu)=g^{-1} \beta_{k}^{\prime} \partial_{u} k+\beta_{\nu}^{\prime} k$, and so the principal part is indeed a diagonal one. The linear operator $A_{1}=\partial_{u}\left(\bar{D} \partial_{u} \Phi\right), D\left(A_{1}\right)=E_{1}$, is a generator of an analytic semigroup on $E_{0}$, and moreover $A_{1} \in \mathcal{M}_{1}\left(E_{0}, E_{1}\right)$ (cf. [8]). Notice that $d_{\alpha} F(\bar{\Phi}, \bar{\alpha})$ belongs to $\mathcal{L}\left(C^{2+\sigma}\left(S^{1}\right), E_{1 / 2}\right)$ and this is why we can write $d_{\Phi} f(\bar{\Phi})$ as the sum $A_{1}+A_{2}$ where $A_{2} \in L\left(E_{1 / 2}, E_{0}\right),\left\|A_{2} \Phi\right\|_{E_{0}} \leq C\|\Phi\|_{E_{1 / 2}} \leq C\|\Phi\|_{E_{0}}^{1 / 2}\|\Phi\|_{E_{1}}^{1 / 2}$ is a relatively bounded linear perturbation of $A_{1}$ with zero relative bound (cf. [8]). Since the class $\mathcal{M}_{1}$ is closed with respect to such perturbations (see [8, Lemma 2.5]), we have $d_{\Phi} f(\bar{\Phi}) \in \mathcal{M}_{1}\left(E_{0}, E_{1}\right)$. The proof of the short time existence of a solution $\Phi$ now follows from [8, Theorem 2.7].

Finally, we will show that the maximal curvature becomes unbounded as $t \rightarrow$ $T_{\max }<\infty$. Suppose to the contrary that $\max _{\Gamma^{t}}|k(., t)| \leq M<\infty$ for any $t \in$ $\left[0, T_{\max }\right)$. According to $[6$, Theorem 3.1], there exists a unique maximal solution $\Gamma:\left[0, T_{\max }^{\prime}\right) \rightarrow \Omega\left(\mathbb{R}^{2}\right)$ satisfying $\Gamma(0)=\Gamma^{0}$ and the geometric equation (1.1). Recall 
that $\Omega\left(\mathbb{R}^{2}\right)$ is the space of $C^{1}$ regular curves in the plane (cf. [6]). Moreover, $\Gamma(t)$ is a $C^{\infty}$ smooth curve for any $t \in\left(0, T_{\max }^{\prime}\right)$ and the maximum of the absolute value of the curvature tends to infinity as $t \rightarrow T_{\max }^{\prime}$. Thus $T_{\max }<T_{\max }^{\prime}$ and therefore the curvature and, subsequently, $\nu$ remain bounded in the $C^{2+\sigma^{\prime}}$ norm on the interval $\left[0, T_{\max }\right]$ for any $\sigma^{\prime} \in(\sigma, 1)$. Applying compactness arguments one sees that the limit $\lim _{t \rightarrow T_{\max }} \Phi(., t)$ exists and remains bounded in the space $E_{1}$ and one can continue the solution $\Phi$ beyond $T_{\max }$, which is a contradiction.

Next we will show how to construct a classical solution $x=x(u, t)$ of the intrinsic heat equation (2.2). Suppose that $\tilde{\Phi}=(\tilde{k}, \tilde{\nu}, \tilde{g})^{T}$ is a classical solution of the system (3.13) existing on the time interval $[0, T]$. Let us construct a flow of plane curves $\Gamma^{t}=\operatorname{Image}(x(., t)), t \in[0, T]$, as follows:

$$
x(u, t)=x^{0}(u)+\int_{0}^{t}(\tilde{\beta} \tilde{\vec{N}}+\tilde{\alpha} \tilde{\vec{T}}) d \tau,
$$

where $\tilde{\vec{N}}=(-\sin \tilde{\nu}, \cos \tilde{\nu})^{T}, \tilde{\vec{T}}=(\cos \tilde{\nu}, \sin \tilde{\nu})^{T}, \tilde{\beta}=\beta(\tilde{k}, \tilde{\nu})$, and $\tilde{\alpha}=\alpha(\tilde{k}, \tilde{\nu}, \tilde{g})$. We claim that $x(u, t)$ is a classical solution of $(2.2)$.

THEOREM 4.2. Assume $\beta$ and $\alpha$ satisfy assumptions of Theorem 4.1. Let $\tilde{\Phi}=$ $(\tilde{k}, \tilde{\nu}, \tilde{g})^{T}$ be a classical solution of (3.13) such that the quantities $\tilde{k}, \tilde{\beta}$, and $\tilde{g}^{-1} \partial_{u} \tilde{\alpha}$ are bounded. Then $x=x(u, t)$ given by (4.5) satisfies $\left|\partial_{u} x\right|=\tilde{g}, k=\tilde{k}, \nu=\tilde{\nu}$, $\vec{N}=\tilde{\vec{N}}, \vec{T}=\tilde{\vec{T}}$, where $k, \nu, \vec{N}, \vec{T}$ represent the curvature, the tangent angle, and the unit normal and tangent vectors of the curve $\Gamma^{t}=\operatorname{Image}(x(., t))$. Moreover, $x \in$ $C\left([0, T] ;\left(C^{2+\sigma}\left(S^{1}\right)\right)^{2}\right) \cap C^{1}\left([0, T] ;\left(C^{\sigma}\left(S^{1}\right)\right)^{2}\right)$ is a classical solution of the intrinsic heat equation (2.2).

Proof. First we prove that $\partial_{u} \tilde{\nu}=\tilde{g} \tilde{k}$ for any classical solution of (3.13). Indeed, if we denote $K=\tilde{k}-\tilde{g}^{-1} \partial_{u} \tilde{\nu}$, then it is easy calculus to verify that $K$ satisfies the linear parabolic equation

$$
\frac{\partial K}{\partial t}=\frac{1}{\tilde{g}} \frac{\partial}{\partial u}\left(\frac{\beta_{k}^{\prime}(\tilde{k}, \tilde{\nu})}{\tilde{g}} \frac{\partial K}{\partial u}\right)-\frac{1}{\tilde{g}} \frac{\partial}{\partial u}\left(\beta_{\nu}^{\prime}(\tilde{k}, \tilde{\nu}) K\right)+\left(\tilde{k} \tilde{\beta}-\frac{1}{\tilde{g}} \frac{\partial \tilde{\alpha}}{\partial u}\right) K .
$$

Moreover, $K(u, 0)=0$ because $\partial_{u} \tilde{\nu}^{0}=\tilde{g}^{0} \tilde{k}^{0}$ (see (3.15)). The term $\tilde{k} \tilde{\beta}-\tilde{g}^{-1} \partial_{u} \tilde{\alpha}$ is assumed to be bounded and therefore we may conclude that $K(u, t)=0$ for any $u \in[0,1], t \in[0, T]$. As $\tilde{g} \tilde{k}=\partial_{u} \tilde{\nu}$ we end up with Frenet's formulae $\partial_{u} \tilde{\vec{T}}=\tilde{g} \tilde{k} \tilde{\vec{N}}$ and $\partial_{u} \tilde{\vec{N}}=-\tilde{g} \tilde{k} \tilde{\vec{T}}$. Similarly as in the proof of the identities (3.1), the equation $\partial_{t} x=\tilde{\beta} \tilde{\vec{N}}+\tilde{\alpha} \tilde{\vec{T}}$ yields $p . \partial_{t} p=\tilde{g}^{2}\left(-k \tilde{\beta}-\tilde{g}^{-1} \partial_{u} \tilde{\alpha}\right)$, where $p=\partial_{u} x$. Thus $\partial_{t}\left(|p|^{2}\right)=$ $2 p . \partial_{t} p=2 \partial_{t}\left(\tilde{g}^{2}\right)$ and therefore $\left|\partial_{u} x\right|=|p|=\tilde{g}$ because $\left|\partial_{u} x^{0}\right|=g^{0}$. Again, using the last two equations in (3.1) we obtain $k=\left(\partial_{u} x \wedge \partial_{u}^{2} x\right) /\left|\partial_{u} x\right|^{3}=\tilde{k}$ and subsequently $\nu=\tilde{\nu}$, which gives us $\vec{N}=\tilde{\vec{N}}, \vec{T}=\tilde{\vec{T}}$. Hence $x=x(u, t)$ obeys (2.4), i.e., $\partial_{t} x=$ $\beta(k, \nu) \vec{N}+\alpha(k, \nu, g) \vec{T}$. Therefore $x$ is a solution of the intrinsic heat equation (2.2). The regularity properties of $x$ follow directly from the regularity of the solution $\tilde{\Phi}$ and (4.5) (see Theorem 4.1).

5. Analysis of the equations for geometric quantities and short time existence of solutions in the degenerate case. The aim of this section is to prove the short time existence of smooth solutions of the curve shortening flow governed by the intrinsic heat equation (2.2). Throughout the rest of the paper we will assume that the normal velocity function $v=\beta(k, \nu)$ has the form

$$
\beta(k, \nu)=\gamma(\nu)|k|^{m-1} k,
$$


where $m>0$ and $\gamma: \mathbb{R} \rightarrow \mathbb{R}^{+}$is a given $C^{\infty}$ smooth anisotropy function satisfying

$$
0<C_{1}^{-1} \leq \gamma(\nu) \leq C_{1}, \quad\left|\gamma_{\nu}^{\prime}(\nu)\right| \leq C_{1} \quad \text { for any } \nu \in \mathbb{R}
$$

where $C_{1}>0$ is a constant.

The assumptions guaranteeing the local existence of classical solutions of a nonlinear curve shortening flow developed by Angenent in $[6,7]$ as well as those of Theorem 4.1 do not directly apply to the case $\beta(k, \nu)=\gamma(\nu)|k|^{m-1} k$ for $m \neq 1$. Recall that these theories require $\beta$ to satisfy (4.2). To use the result established in Theorem 4.1 we must go through a regularization argument. A similar technique was applied in the paper by Angenent, Sapiro, and Tannenbaum [12] for the case of an isotropic function $\beta(k)=k^{1 / 3}$. In what follows, we will slightly modify their approach for the more general anisotropic power-like function $\beta(k, \nu)$ and for the case when the curvature equation involves a nontrivial tangential velocity term $\alpha$.

Henceforth, we denote by $C_{i}, M_{i}$ any generic positive constant independent on the regularization parameter $0<\varepsilon \leq 1$. Constants $M_{i}$ may also depend on the initial curve $\Gamma^{0}$. We make the following regularization assumption on the function $\beta$ :

There is a family of nondecreasing $C^{\infty}$ functions $\beta^{\varepsilon}, 0<\varepsilon \leq 1$, such that

(i) $\beta^{\varepsilon}(k, \nu) \rightarrow \beta^{0}(k, \nu)=\beta(k, \nu)$ as $\varepsilon \rightarrow 0^{+}$locally uniformly with respect to $(k, \nu) \in \mathbb{R}^{2}$

(ii) $\left|\beta^{\varepsilon}(k, \nu)\right| \leq C_{2}\left(1+|k|^{m}\right)$ for any $k, \nu \in \mathbb{R}$;

(iii) there exist constants $\lambda_{ \pm}^{\varepsilon}=\lambda_{ \pm}^{\varepsilon}\left(M_{1}\right)>0$ such that $\lambda_{-}^{\varepsilon} \leq \beta_{k}^{\varepsilon \prime}(k, \nu) \leq \lambda_{+}^{\varepsilon}$;

(iv) $\left|\beta_{k}^{\varepsilon \prime}(k, \nu) k^{4}\left(\beta^{\varepsilon}(k, \nu)\right)^{2}\right|+\frac{\left|\beta_{\nu}^{\varepsilon}(k, \nu)\right|^{2}}{\beta_{k}^{\varepsilon}(k, \nu)} \leq C_{3}\left(M_{1}\right)$ for any $|k| \leq M_{1}$ and $\nu \in \mathbb{R}$.

It is easy to verify that the regularization family $\left(\beta^{\varepsilon}\right)$ defined as

$$
\begin{aligned}
& \beta^{\varepsilon}(k, \nu)=m \gamma(\nu) \int_{0}^{k}\left(\varepsilon^{2}+\xi^{2}\right)^{\frac{m-1}{2}} d \xi \quad \text { if } 0<m \leq 1, \\
& \beta^{\varepsilon}(k, \nu)=\beta(k, \nu)+\varepsilon k \quad \text { if } m>1
\end{aligned}
$$

satisfies the above assumptions (i)-(iv) with constants $\lambda_{ \pm}^{\varepsilon}>0$ given by

$$
\begin{array}{ll}
\lambda_{-}^{\varepsilon}=\delta, & \lambda_{+}^{\varepsilon}=C_{1} m \varepsilon^{m-1} \quad \text { if } 0<m \leq 1, \\
\lambda_{-}^{\varepsilon}=\varepsilon, & \lambda_{+}^{\varepsilon}=1+\max _{|k| \leq M_{1}} \beta_{k}^{\prime}(k, \nu) \quad \text { if } m>1,
\end{array}
$$

where $\delta>0$ is a constant independent of $0<\varepsilon \leq 1$. Furthermore,

$$
0 \leq \frac{\beta^{\varepsilon}(k, \nu)}{k} \leq \max \left(1, m^{-1}\right) \beta_{k}^{\varepsilon \prime} \quad \text { for any } k, \nu \in \mathbb{R} \text { and } 0<\varepsilon \leq 1 .
$$

Let us emphasize the fact that the tangential velocity $\alpha$ may also depend on the regularization parameter $\varepsilon$, i.e., $\alpha=\alpha^{\varepsilon}$. For instance, $\alpha^{\varepsilon}$ may depend on $k$ and $\beta^{\varepsilon}=\beta^{\varepsilon}(k, \nu)$. Concerning the structural properties of $\alpha^{\varepsilon}$ we make the following hypotheses:

$$
\sup _{\Phi \in B_{1 / 2}}\left\{\left|\alpha^{\varepsilon}\right|+\left|\partial_{s} \alpha^{\varepsilon}\right| ; \quad \alpha^{\varepsilon}=\alpha^{\varepsilon}(\Phi), 0 \leq \varepsilon \leq 1\right\}<\infty
$$

for any set $B_{1 / 2}=\left\{(k, \nu, g)^{T} \in \mathcal{O}_{1 / 2},|k| \leq M_{1}\right\}$ and

$$
\left\|\alpha^{\varepsilon}(k, \nu, g)\right\|_{C^{2}\left(S^{1}\right)} \leq C\left(1+\|g\|_{C^{1}\left(S^{1}\right)}+\left\|\beta^{\varepsilon}(k, \nu)\right\|_{C^{1}\left(S^{1}\right)}^{q}\right)
$$


for any $\Phi=(k, \nu, g)^{T} \in B_{1 / 2}$, where $C=C\left(M_{1}\right)>0$ is a constant and $1 \leq q<\frac{4}{3}$. In section 6 we will show how to construct a so-called tangential velocity preserving the relative local length satisfying the above hypotheses.

Let $\Gamma^{0}$ be a smooth initial curve such that $\Phi^{0}=\left(k^{0}, \nu^{0}, g^{0}\right)^{T} \in \mathcal{O}_{1} \subset E_{1}$. By $\Phi_{\varepsilon}=\left(k_{\varepsilon}, \nu_{\varepsilon}, g_{\varepsilon}\right)^{T}$ we denote the classical solution of the governing system of equations (3.13) with $\beta=\beta^{\varepsilon}$ and $\alpha=\alpha^{\varepsilon}$. The short time existence of $\Phi_{\varepsilon}$ has been justified by Theorem 4.1 for any $0<\varepsilon \leq 1$. From (4.5) and Theorem 4.2 we furthermore know that the function

$$
x_{\varepsilon}(u, t)=x^{0}(u)+\int_{0}^{t}\left(\beta^{\varepsilon} \vec{N}_{\varepsilon}+\alpha^{\varepsilon} \vec{T}_{\varepsilon}\right) d \tau
$$

is a classical solution of the intrinsic heat equation (2.2) for any $0<\varepsilon \leq 1$.

First we will show that the maximum of $|k|$ remains bounded in a short time interval $[0, T]$ and the parameterization of the curve $\Gamma^{t}$ is regular.

LEMma 5.1. Suppose that the regularization assumptions (i), (ii) are satisfied. Then there exist constants $T>0$ and $M_{1}>0$ such that

$$
\max _{\Gamma^{t}}\left|k_{\varepsilon}(., t)\right| \leq M_{1} \quad \text { for any } t \in[0, T] \text { and } \varepsilon \in(0,1] .
$$

If $\alpha^{\varepsilon}$ satisfies (5.4), then there are constants $g_{ \pm}>0$ such that

$$
0<g_{-}<g_{\varepsilon}(u, t)<g_{+}<\infty \text { for any }(u, t) \in Q_{T} \text { and } \varepsilon \in(0,1] \text {. }
$$

Proof. The proof of the first part is essentially the same as that of [12, Theorem 6.2]. Indeed, as $\partial_{t} k_{\varepsilon}=\partial_{s}^{2} \beta^{\varepsilon}+\alpha^{\varepsilon} \partial_{s} k_{\varepsilon}+k_{\varepsilon}^{2} \beta^{\varepsilon}$, then by applying a maximum principle argument we get $\partial_{t}\left(\max _{\Gamma_{\varepsilon}^{t}}\left|k_{\varepsilon}(., t)\right|\right) \leq F^{\varepsilon}\left(\max _{\Gamma_{\varepsilon}^{t}}\left|k_{\varepsilon}(., t)\right|\right)$, where $F^{\varepsilon}(k)=\max _{\nu} k^{2}\left|\beta^{\varepsilon}(k, \nu)\right| \leq C_{2} k^{2}\left(1+|k|^{m}\right)$ for any $0<\varepsilon \leq 1$. Solving this differential inequality we conclude the proof of the bound for the total variation of the curvature. To prove estimates on $g$ we integrate the third equation in (3.14) with respect to time. We obtain $g_{\varepsilon}(u, t)=g^{0}(u) \exp \left(\int_{0}^{t}\left(-k_{\varepsilon} \beta^{\varepsilon}+g_{\varepsilon}^{-1} \partial_{u} \alpha^{\varepsilon}\right) d \tau\right)$, where $\beta^{\varepsilon}=\beta^{\varepsilon}\left(k_{\varepsilon}, \nu_{\varepsilon}\right)$. The proof now follows from the fact that both $k_{\varepsilon} \beta^{\varepsilon}$ and $g_{\varepsilon}^{-1} \partial_{u} \alpha^{\varepsilon}=\partial_{s} \alpha^{\varepsilon}$ are bounded for $|k| \leq M_{1}$ and $0<g^{0}<\infty$ uniformly with respect to $\varepsilon \in(0,1]$.

In the next lemma we analyze the degenerate case when $1<m \leq 2$. It is a key technical tool in order to establish some a priori estimates needed in the proof of short time existence of a solution in this degenerate case. Interestingly enough, a new geometric assumption on the initial curve is needed.

Lemma 5.2. Assume $1<m \leq 2$. Suppose that the initial curve $\Gamma^{0}$ satisfies

$$
\int_{\Gamma^{0}} \frac{k^{0}}{\beta\left(k^{0}, \nu^{0}\right)} d s<\infty
$$

Then there exists a constant $M_{2}>0$ such that

$$
\max _{t \in[0, T]} \int_{\Gamma^{t}} \frac{k_{\varepsilon}}{\beta^{\varepsilon}\left(k_{\varepsilon}, \nu_{\varepsilon}\right)} d s+\int_{0}^{T} \int_{\Gamma^{t}}\left|\partial_{s} k_{\varepsilon}\right|^{2} d s \leq M_{2} \quad \text { for any } 0<\varepsilon \leq 1 .
$$

Proof. Denote $v=\beta^{\varepsilon}\left(k_{\varepsilon}, \nu_{\varepsilon}\right), k=k_{\varepsilon}$, and $\nu=\nu_{\varepsilon}$. By using (3.4), the curvature equation (3.2) and the velocity equation (3.10), we obtain

$$
\frac{d}{d t} \int_{\Gamma^{t}} \frac{k}{v} d s=\int_{\Gamma^{t}} \frac{\partial}{\partial t}\left(\frac{k}{v}\right)+\frac{k}{v}\left(-k v+\partial_{s} \alpha\right) d s
$$




$$
\begin{aligned}
= & \int_{\Gamma^{t}}\left(\frac{1}{v}-\frac{k \beta_{k}^{\varepsilon \prime}}{v^{2}}\right)\left(\partial_{s}^{2} v+\alpha \partial_{s} k+k^{2} v\right) \\
& -\frac{k \beta_{\nu}^{\varepsilon \prime}}{v^{2}}\left(\partial_{s} v+\alpha k\right)-k^{2}+\frac{k}{v} \partial_{s} \alpha d s \\
= & \int_{\Gamma^{t}}\left(\frac{1}{v}-\frac{k \beta_{k}^{\varepsilon \prime}}{v^{2}}\right) \partial_{s}^{2} v-\frac{k^{3} \beta_{k}^{\varepsilon \prime}}{v}-\frac{k \beta_{\nu}^{\varepsilon \prime}}{v^{2}} \partial_{s} v d s
\end{aligned}
$$

because of the identity $0=\int_{\Gamma^{t}} \partial_{s}\left(\frac{\alpha k}{v}\right) d s=\int_{\Gamma^{t}} \frac{k}{v} \partial_{s} \alpha+\frac{\alpha}{v} \partial_{s} k-\frac{\alpha k}{v^{2}} \partial_{s} v d s$, (3.3), and (3.11). Recall that $\beta_{k}^{\varepsilon \prime}=\beta_{k}^{\prime}+\varepsilon=\frac{m \beta}{k}+\varepsilon$ and therefore

$$
\frac{k \beta_{k}^{\varepsilon \prime}}{v}=m+\varepsilon(1-m) \frac{k}{v}
$$

Plugging the above expression into (5.8) and integration by parts yield the identity

$$
\begin{gathered}
\frac{d}{d t} \int_{\Gamma^{t}} \frac{k}{v} d s+(m-1) \int_{\Gamma^{t}} \frac{1}{v^{2}}\left(\left|\partial_{s} v\right|^{2}+\varepsilon \partial_{s} v \partial_{s} k-2 \varepsilon \frac{k}{v}\left|\partial_{s} v\right|^{2}\right) d s \\
=-m \int_{\Gamma^{t}} k^{2} d s+\varepsilon(m-1) \int_{\Gamma^{t}} \frac{k^{3}}{v} d s-\int_{\Gamma^{t}} \frac{k \beta_{\nu}^{\varepsilon \prime}}{v^{2}} \partial_{s} v d s
\end{gathered}
$$

It follows from (3.11) that $\partial_{s} k=\left(\partial_{s} v-\beta_{\nu}^{\varepsilon \prime} k\right) / \beta_{k}^{\varepsilon \prime}$. Thus

$$
\begin{aligned}
& \frac{d}{d t} \int_{\Gamma^{t}} \frac{k}{v} d s+(m-1) \int_{\Gamma^{t}}\left|\frac{1}{v} \partial_{s} v\right|^{2}\left(1+\frac{\varepsilon}{\beta_{k}^{\varepsilon \prime}}-2 \varepsilon \frac{k}{v}\right) d s \\
& \quad=-m \int_{\Gamma^{t}} k^{2}\left(1-\frac{\varepsilon k}{v}\right) d s-\varepsilon \int_{\Gamma^{t}} \frac{k^{3}}{v} d s+\int_{\Gamma^{t}} \frac{k \beta_{\nu}^{\varepsilon \prime}}{v^{2}} \partial_{s} v\left(\frac{\varepsilon(m-1)}{\beta_{k}^{\varepsilon \prime}}-1\right) d s \\
& \quad \leq \int_{\Gamma^{t}} \frac{\gamma^{\prime}(\nu)}{\gamma(\nu)} \frac{k}{v} \partial_{s} v\left(\frac{\varepsilon(m-1)}{\beta_{k}^{\varepsilon \prime}}-1\right) d s \leq m C_{1}^{2} \int_{\Gamma^{t}}\left|\frac{k}{v} \partial_{s} v\right| d s
\end{aligned}
$$

because of the inequalities $\left|\frac{\beta_{\nu}^{\varepsilon^{\prime}}}{v}\right|=\left|\frac{\gamma^{\prime}(\nu)}{\gamma(\nu)}\right| \leq C_{1}^{2}, 0 \leq \frac{\varepsilon k}{v} \leq 1$, and $0<\frac{\varepsilon}{\beta_{k}^{\varepsilon^{\prime}}} \leq 1$. Let us consider the auxiliary function $\phi$ defined as follows:

$$
\phi(k)=\frac{1}{k^{2}}\left(1+\frac{\varepsilon}{\beta_{k}^{\varepsilon \prime}}-\frac{2 \varepsilon k}{v}\right)=\frac{1}{k^{2}}\left(1+\frac{\varepsilon}{m \gamma(\nu)|k|^{m-1}+\varepsilon}-\frac{2 \varepsilon}{\gamma(\nu)|k|^{m-1}+\varepsilon}\right) .
$$

It is easy calculus to verify that if $1<m \leq 2$ then there exists a constant $M_{3}>0$ independent of $0<\varepsilon \leq 1$ and such that $\inf _{|k| \leq M_{1}} \phi(k) \geq M_{3}$. Using the CauchySchwarz inequality we get

$$
\begin{gathered}
\frac{d}{d t} \int_{\Gamma^{t}} \frac{k}{v} d s+M_{3}(m-1) \int_{\Gamma^{t}}\left|\frac{k}{v} \partial_{s} v\right|^{2} d s \leq m C_{1}^{2} \int_{\Gamma^{t}}\left|\frac{k}{v} \partial_{s} v\right| d s \\
\leq \frac{m^{2} C_{1}^{4}}{2(m-1) M_{3}} L^{t}+\frac{M_{3}(m-1)}{2} \int_{\Gamma^{t}}\left|\frac{k}{v} \partial_{s} v\right|^{2} d s
\end{gathered}
$$

and so

$$
\frac{d}{d t} \int_{\Gamma^{t}} \frac{k}{v} d s+\frac{M_{3}(m-1)}{2} \int_{\Gamma^{t}}\left|\frac{k}{v} \partial_{s} v\right|^{2} d s \leq \frac{m^{2} C_{1}^{4}}{2(m-1) M_{3}} L^{0}
$$


Integrating the above inequality over the interval $[0, T]$, taking into account the inequality

$$
\left|\frac{k}{v} \partial_{s} v\right|=\left|\left(m+(1-m) \frac{\varepsilon k}{v}\right) \partial_{s} k+\frac{\gamma^{\prime}(\nu)}{\gamma(\nu)} k^{2}\right| \geq\left|\partial_{s} k\right|-C_{1}^{2} M_{1}^{2}
$$

and the initial time inequality $\int_{\Gamma^{0}} \frac{k}{v} d s=\int_{\Gamma^{0}} \frac{k^{0}}{\beta^{\varepsilon}\left(k^{0}, \nu^{0}\right)} d s \leq \int_{\Gamma^{0}} \frac{k^{0}}{\beta\left(k^{0}, \nu^{0}\right)} d s<\infty$ we finally obtain the estimate (5.7).

Remark 5.1. The assumption (5.6) seems to be quite restrictive. Note that it is fulfilled in the case when the initial curve $\Gamma^{0}$ is strictly convex or in the case of a nonconvex smooth curve whose inflection points have at most $\left(2+\frac{1}{m-1}\right)$-order contact with their tangents. As an example one can consider the Bernoulli lemniscate $\left(x^{2}+y^{2}\right)^{2}=4 x y$ having the third-order contact with its tangents at the origin. In this example the assumption (5.6) is satisfied iff $1<m<2$.

Remark 5.2. It would be of interest to know whether the power $m=2$ is an optimal value. It follows from recent results due to Andrews [4] that for higher powers of $m$ the curve $\Gamma^{t}$ need not be sufficiently smooth in the vicinity of a point where the curvature vanishes.

Lemma 5.3. For any $t \in(0, T)$ we have

$$
\frac{d}{d t} X_{p}(t) \leq-\int_{\Gamma^{t}} \beta_{k}^{\varepsilon \prime}\left|\partial_{s}\left(w^{p / 2}\right)\right|^{2} d s+M_{4} p^{2}\left(1+X_{p}(t)\right),
$$

where $X_{p}(t)=\int_{\Gamma^{t}}|w|^{p} d s=\int_{0}^{1}|w|^{p}\left|\partial_{u} x_{\varepsilon}\right| d u$ and $w=\partial_{s} \beta^{\varepsilon}\left(k_{\varepsilon}, \nu_{\varepsilon}\right), p \geq 1$.

Proof. Denote $k=k_{\varepsilon}, \nu=\nu^{\varepsilon}$. Applying the local length equation (3.4) and the equation for the gradient of velocity (3.12) we obtain

$$
\begin{aligned}
\frac{d}{d t} X_{p}(t)= & \int_{0}^{1}\left(\partial_{t}\left(|w|^{p}\right)\left|\partial_{u} x\right|+|w|^{p}\left(-\left|\partial_{u} x\right| k v+\partial_{u} \alpha^{\varepsilon}\right)\right) d u \\
= & \int_{\Gamma^{t}}\left(p|w|^{p-2} w \partial_{t} w+|w|^{p}\left(-k v+\partial_{s} \alpha^{\varepsilon}\right)\right) d s \\
= & \int_{\Gamma^{t}}\left(p|w|^{p-2} w\left[\partial_{s}\left(\beta_{k}^{\varepsilon \prime} \partial_{s} w+\beta_{k}^{\varepsilon \prime} k^{2} v+\beta_{\nu}^{\varepsilon \prime} w\right)+\alpha^{\varepsilon} \partial_{s} w+k v w\right]\right. \\
& \left.+|w|^{p}\left(-k v+\partial_{s} \alpha^{\varepsilon}\right)\right) d s \\
= & -p(p-1) \int_{\Gamma^{t}}|w|^{p-2}\left[\beta_{k}^{\varepsilon \prime}\left|\partial_{s} w\right|^{2}+\beta_{k}^{\varepsilon} k^{2} v \partial_{s} w+\beta_{\nu}^{\varepsilon \prime} w \partial_{s} w\right] d s \\
& \quad+(p-1) \int_{\Gamma^{t}}|w|^{p} k v d s
\end{aligned}
$$

because $0=\int_{\Gamma^{t}} \partial_{s}\left(|w|^{p} \alpha^{\varepsilon}\right)=\int_{\Gamma^{t}} p|w|^{p-2} w \partial_{s} w \alpha^{\varepsilon}+|w|^{p} \partial_{s} \alpha^{\varepsilon} d s$. Notice that the tangential velocity term $\alpha^{\varepsilon}$ is involved neither in the expression for $X_{p}$ nor in $\frac{d}{d t} X_{p}$.

Applying the Cauchy-Schwarz inequality we get

$$
\left|\beta_{k}^{\varepsilon \prime} k^{2} v \partial_{s} w+\beta_{\nu}^{\varepsilon \prime} w \partial_{s} w\right| \leq \beta_{k}^{\varepsilon \prime} k^{4} v^{2}+\beta_{k}^{\varepsilon \prime} \frac{\left|\partial_{s} w\right|^{2}}{4}+\frac{\left|\beta_{\nu}^{\varepsilon \prime}\right|^{2}}{\beta_{k}^{\varepsilon \prime}}|w|^{2}+\beta_{k}^{\varepsilon \prime} \frac{\left|\partial_{s} w\right|^{2}}{4}
$$

and therefore

$$
\frac{d}{d t} X_{p}(t)=-\frac{p(p-1)}{2} \int_{\Gamma^{t}} \beta_{k}^{\varepsilon \prime}|w|^{p-2}\left|\partial_{s} w\right|^{2} d s
$$




$$
\begin{aligned}
& +p(p-1) \int_{\Gamma^{t}}|w|^{p-2} \beta_{k}^{\varepsilon \prime} k^{4} v^{2}+|w|^{p} \frac{\left|\beta_{\nu}^{\varepsilon \prime}\right|^{2}}{\beta_{k}^{\varepsilon \prime}} d s+(p-1) \int_{\Gamma^{t}}|w|^{p} k v d s \\
\leq & -\int_{\Gamma^{t}} \beta_{k}^{\varepsilon \prime}\left|\partial_{s}\left(w^{p / 2}\right)\right|^{2} d s \\
& +C_{3} p(p-1)\left(X_{p-2}(t)+X_{p}(t)\right)+(p-1) M_{1}^{2} X_{p}(t)
\end{aligned}
$$

because $|w|^{p-2}\left|\partial_{s} w\right|^{2}=\frac{4}{p^{2}}\left|\partial_{s}\left(w^{p / 2}\right)\right|^{2}$ and $2 p(p-1) / p^{2} \geq 1$ for any $p \geq 2$. Since $X_{p-2}(t)=\int_{\Gamma^{t}}|w|^{p-2} \leq \int_{\Gamma^{t}}\left(1+|w|^{p}\right) \leq L^{t}+X_{p}(t)$ for any $p \geq 2$ and $L^{t} \leq L^{0}$ (see (3.5)) we finally obtain the inequality (5.9) with a constant $M_{4}>0$ independent of $0<\varepsilon \leq 1$ and $p \geq 2$.

LEMMA 5.4. Suppose that $0<m \leq 2$. If $1<m \leq 2$ we additionally suppose that the initial curve $\Gamma^{0}$ satisfies the condition (5.6). Then there is a constant $M_{7}>0$ such that

$$
\begin{aligned}
& \text { if } 0<m \leq 1 \text { then } \max _{\Gamma^{t}}\left|\partial_{s} \beta^{\varepsilon}\left(k_{\varepsilon}, \nu_{\varepsilon}\right)\right| \leq M_{7} t^{-\frac{3}{4}} ; \\
& \text { if } 1<m \leq 2 \text { then } \max _{\Gamma^{t}}\left|\partial_{s} \beta^{\varepsilon}\left(k_{\varepsilon}, \nu_{\varepsilon}\right)\right| \leq M_{7} t^{-\frac{1}{2}}
\end{aligned}
$$

for any $0<\varepsilon \leq 1$ and $0<t \leq T$.

Proof. The key idea behind the proof of this estimate is a modification of the well-known Nash-Moser iterative technique adopted to the flow of plane curves. It is similar, in spirit and technique, to that of [12, Chapter 6], which has been applied in the case of the affine scaling parameterization, i.e., $\beta(k)=k^{1 / 3}$. By using the differential inequality (5.9) we will show that $\|w\|_{p}=X_{p}^{1 / p}(t)$ is bounded uniformly with respect to $p \geq 2$ and $0<\varepsilon \leq 1$, yielding the desired $L^{\infty}$ estimate on $\partial_{s} \beta^{\varepsilon}\left(k_{\varepsilon}, \nu_{\varepsilon}\right)$.

Let us consider the case $0<m \leq 1$. First, we will prove an estimate for $X_{2}(t)$. By (5.2) we have $\beta_{k}^{\varepsilon \prime} \geq \delta>0$ and $\beta^{\varepsilon} \leq M_{5}$. Then

$$
\begin{aligned}
X_{2} & =\int_{\Gamma^{t}}|w|^{2}=\int_{\Gamma^{t}}\left(\partial_{s} v\right)^{2}=-\int_{\Gamma^{t}} v \partial_{s}^{2} v=-\int_{\Gamma^{t}} \beta^{\varepsilon} \partial_{s} w \\
& \leq \frac{M_{5}}{\delta} \int_{\Gamma^{t}} \sqrt{\beta_{k}^{\varepsilon \prime}}\left|\partial_{s} w\right| \leq \frac{M_{5}\left(L^{t}\right)^{\frac{1}{2}}}{\delta}\left(\int_{\Gamma^{t}} \beta_{k}^{\varepsilon \prime}\left|\partial_{s} w\right|^{2}\right)^{\frac{1}{2}} .
\end{aligned}
$$

According to (5.9), $X_{2}$ is a solution of the differential inequality

$$
\frac{d X_{2}}{d t} \leq-\frac{1}{M^{2}} X_{2}^{2}+4 M_{4}\left(1+X_{2}\right)
$$

where $M=M_{5}\left(L^{0}\right)^{\frac{1}{2}} / \delta$. By solving the above differential inequality we obtain $X_{2}(t) \leq A_{2}^{2} t^{-1}$, where $A_{2}$ is a constant depending only on $m, \Gamma^{0}$, and $T$.

Let $p \geq 2$. As $w=\partial_{s} v$ there must be a point at $\Gamma^{t}$ where $w^{p / 2}$ vanishes. From the interpolation inequality [12, Proposition $6.1,(25)]$ we infer

$$
X_{p}=\int_{\Gamma^{t}}|w|^{p} \leq\left(\int_{\Gamma^{t}}|w|^{\frac{p}{2}}\right)^{\frac{4}{3}}\left(\int_{\Gamma^{t}}\left|\partial_{s}\left(w^{\frac{p}{2}}\right)\right|^{2}\right)^{\frac{1}{3}} \leq X_{\frac{p}{2}}^{\frac{4}{3}}\left(\frac{1}{\delta} \int_{\Gamma^{t}} \beta_{k}^{\varepsilon \prime}\left|\partial_{s}\left(w^{\frac{p}{2}}\right)\right|^{2}\right)^{\frac{1}{3}} .
$$

Let us consider the case $1<m \leq 2$. Again, we begin with an estimate for $X_{2}(t)$. For $1<m$ the derivative $\beta_{k}^{\varepsilon \prime}$ is bounded uniformly with respect to $0<\varepsilon \leq 1$. Since $|k| \leq M_{1}$ we have $X_{2}(0)=\int_{\Gamma^{0}}\left|\partial_{s} \beta^{\varepsilon}\right|^{2} \leq M \int_{\Gamma^{0}}\left(1+\left|\partial_{s} k^{0}\right|^{2}\right)<\infty$. Integrating the differential inequality (5.9) we obtain $X_{2}(t) \leq 1+X_{2}(t) \leq\left(1+X_{2}(0)\right) \exp \left(4 M_{4} t\right) \leq A_{2}^{2}$ for any $0 \leq t \leq T$, where $A_{2}>0$ is a constant. By using the Cauchy-Schwarz inequality we obtain

$$
\sup _{\Gamma^{t}}|w|^{\frac{p}{2}} \leq \int_{\Gamma^{t}}\left|\partial_{s}\left(w^{\frac{p}{2}}\right)\right| \leq\left(\int_{\Gamma^{t}} \frac{1}{\beta_{k}^{\varepsilon \prime}}\right)^{\frac{1}{2}}\left(\int_{\Gamma^{t}} \beta_{k}^{\varepsilon \prime}\left|\partial_{s}\left(w^{\frac{p}{2}}\right)\right|^{2}\right)^{\frac{1}{2}} .
$$


According to (5.3) and Lemma 5.2 we have $\left(\int_{\Gamma^{t}} \frac{1}{\beta_{k}^{\varepsilon^{\prime}}}\right)^{\frac{1}{2}} \leq M_{6}$ and hence

$$
X_{p}=\int_{\Gamma^{t}}|w|^{p} \leq \sup _{\Gamma^{t}}|w|^{\frac{p}{2}} X_{\frac{p}{2}} \leq M_{6} X_{\frac{p}{2}}\left(\int_{\Gamma^{t}} \beta_{k}^{\varepsilon \prime}\left|\partial_{s}\left(w^{\frac{p}{2}}\right)\right|^{2}\right)^{\frac{1}{2}} .
$$

In both cases, taking into account (5.9) we end up with a differential inequality

$$
\frac{d}{d t} X_{p}(t) \leq-\hat{\delta} \frac{X_{p}^{L}(t)}{X_{p / 2}^{K}(t)}+M p^{2}\left(1+X_{p}(t)\right)
$$

for any $t \in(0, T], p \geq 2$, and $0<\varepsilon \leq 1$, where $(L, K)=(3,4)$ if $0<m \leq 1$, $(L, K)=(2,2)$ if $1<m \leq 2$, and $\hat{\delta}, M>0$ are constants independent of $0<\varepsilon \leq 1$ and $p \geq 2$. In the case $(L, K)=(3,4)$ this is exactly the same differential inequality as that of $[12$, eq. (2.4)]. Following the iterative method of supersolutions to the differential inequality (5.10) presented in [12, Chapter 6], given a couple $(L, K)$ such that $K=2(L-1), L>1$, one can prove the existence of a bounded sequence $\left(A_{k}\right)$, $0<A_{k} \leq M_{7}$, such that

$$
X_{p_{k}}(t) \leq A_{k}^{p_{k}} t^{-\alpha_{k} p_{k}}
$$

for any $p_{k}=2^{k+1}, k \geq 0$, where $\alpha_{0}=\frac{1}{2}$ for $0<m \leq 1, \alpha_{0}=0$ for $1<m \leq 2$ and

$$
\alpha_{k+1}=\alpha_{k}+\frac{1}{(L-1)} 2^{-k-2}=\alpha_{0}+\frac{1}{L-1} \sum_{l=0}^{k} 2^{-l-2} \rightarrow \alpha_{0}+\frac{1}{2(L-1)}
$$

as $k \rightarrow \infty$. This yields the estimate

$$
\sup _{\Gamma^{t}}\left|\partial_{s} \beta^{\varepsilon}\right|=\lim _{k \rightarrow \infty} X_{p_{k}}^{\frac{1}{p_{k}}}(t) \leq M_{7} t^{-\left(\alpha_{0}+\frac{1}{2(L-1)}\right)}
$$

for any $t \in(0, T]$ and $0<\varepsilon \leq 1$. Since $\alpha_{0}=\frac{1}{2}, L=3$ for $0<m \leq 1$ and $\alpha_{0}=0, L=2$ for $1<m \leq 2$, the proof of the lemma follows.

Summarizing all the previous results we conclude the following a priori estimates.

Lemma 5.5. Assume $0<m \leq 2$. Let $\Phi_{\varepsilon}=\left(k_{\varepsilon}, \nu_{\varepsilon}, g_{\varepsilon}\right)^{T}$ be a classical solution of (3.13) existing on the interval $I=[0, T]$ and satisfying the initial condition $\Phi^{0} \in$ $\mathcal{O}_{1} \subset E_{1}$. If $1<m \leq 2$ we furthermore assume that the initial curve $\Gamma^{0}$ satisfies the condition (5.6). If the tangential velocity $\alpha^{\varepsilon}$ satisfies the condition (5.4), then

(1) $k_{\varepsilon}, \beta^{\varepsilon}, t^{\frac{3}{4}} \partial_{u} \beta^{\varepsilon} \in L^{\infty}\left(Q_{T}\right)$;

(2) $g_{\varepsilon}, g_{\varepsilon}^{-1} \in W^{1, \infty}\left(I, L^{\infty}\left(S^{1}\right)\right)$;

(3) $\partial_{u} \nu_{\varepsilon}, t^{\frac{3}{4}} \partial_{t} \nu_{\varepsilon} \in L^{\infty}\left(Q_{T}\right)$

(4) $x_{\varepsilon} \in\left(W^{1, \infty}\left(Q_{T}\right)\right)^{2}$;

and if, in addition, $\alpha^{\varepsilon}$ satisfies the condition (5.5), then

(5) $g_{\varepsilon}, g_{\varepsilon}^{-1} \in W^{1, \infty}\left(Q_{T}\right)$ and $\partial_{u} x_{\varepsilon} \in\left(W^{1, \infty}\left(Q_{T}\right)\right)^{2}$

and their corresponding norms are bounded independently of $0<\varepsilon \leq 1$.

Proof. The statement (1) is an immediate consequence of Lemmas 5.1 and 5.4 and the assumption (ii) made on the regularization $\beta^{\varepsilon}$. Since $\partial_{t} g_{\varepsilon}=-g_{\varepsilon} k_{\varepsilon} \beta^{\varepsilon}+\partial_{u} \alpha^{\varepsilon}$ and $\partial_{t} g_{\varepsilon}^{-1}=-g_{\varepsilon}^{-2} \partial_{t} g_{\varepsilon}$ the statement (2) follows from (1), Lemma 5.1, and the assumption (5.4). The bounds for $\nu_{\varepsilon}$ follow from the identities $\partial_{u} \nu_{\varepsilon}=g_{\varepsilon} k_{\varepsilon}, \partial_{t} \nu_{\varepsilon}=\partial_{s} \beta^{\varepsilon}+\alpha^{\varepsilon} k_{\varepsilon}$ (see (3.8), (3.9)). As $\partial_{t} x_{\varepsilon}=\beta^{\varepsilon} \vec{N}_{\varepsilon}+\alpha^{\varepsilon} \vec{T}_{\varepsilon}, \partial_{u} x_{\varepsilon}=g_{\varepsilon} \vec{T}_{\varepsilon}$, and $\beta^{\varepsilon}, \alpha^{\varepsilon}, g_{\varepsilon} \in L^{\infty}\left(S^{1}\right)$ we conclude the statement (4). Let us assume $\alpha^{\varepsilon}$ satisfies the condition (5.5). By 
integrating the third equation in (3.13) we obtain $g_{\varepsilon}(., t)=g^{0}(.) \exp \left(\int_{0}^{t}\left(-k_{\varepsilon} \beta^{\varepsilon}+\right.\right.$ $\left.\left.g_{\varepsilon}^{-1} \partial_{u} \alpha^{\varepsilon}\right) d \tau\right)$. Furthermore,

$$
\partial_{u}\left(k_{\varepsilon} \beta^{\varepsilon}\right)=k_{\varepsilon} \partial_{u} \beta^{\varepsilon}+\beta^{\varepsilon} \partial_{u} k_{\varepsilon}=\left(k_{\varepsilon}+\frac{\beta^{\varepsilon}}{\beta_{k}^{\varepsilon \prime}}\right) \partial_{u} \beta^{\varepsilon}-\frac{\beta^{\varepsilon} \beta_{\nu}^{\varepsilon \prime}}{\beta_{k}^{\varepsilon \prime}} g_{\varepsilon} k_{\varepsilon} .
$$

With regard to (1) and the regularization assumption made on $\beta^{\varepsilon}$, we can conclude $\left\|k_{\varepsilon} \beta^{\varepsilon}\right\|_{C^{1}} \leq M\left\|\beta^{\varepsilon}\right\|_{C^{1}}$. Taking into account the condition (5.5) and Lemma 5.4 we obtain the estimate

$$
\begin{aligned}
\left\|g_{\varepsilon}(., t)\right\|_{C^{1}} & \leq M\left(1+\int_{0}^{t}\left(\left\|k_{\varepsilon} \beta^{\varepsilon}\right\|_{C^{1}}+\left\|\alpha^{\varepsilon}\right\|_{C^{2}}+\left\|g_{\varepsilon}(., \tau)\right\|_{C^{1}}\right) d \tau\right) \\
& \leq M\left(1+\int_{0}^{t}\left\|\beta^{\varepsilon}\right\|_{C^{1}} d \tau+\int_{0}^{t}\left\|g_{\varepsilon}(., \tau)\right\|_{C^{1}} d \tau\right) \\
& \leq M\left(1+\int_{0}^{t}\left\|g_{\varepsilon}(., \tau)\right\|_{C^{1}} d \tau\right)
\end{aligned}
$$

for $t \in[0, T]$ and $0<\varepsilon \leq 1$. Hence the $L^{\infty}$ bounds for $\partial_{u} g_{\varepsilon}$ and $\partial_{u} g_{\varepsilon}^{-1}=-g_{\varepsilon}^{-2} \partial_{u} g_{\varepsilon}$ follow from Gronwall's lemma. The $L^{\infty}$ bounds for $\partial_{u}^{2} x_{\varepsilon}$ and $\partial_{t} \partial_{u} x_{\varepsilon}$ now follow from the identities $\partial_{u}^{2} x_{\varepsilon}=\partial_{u}\left(g_{\varepsilon} T_{\varepsilon}\right)=\partial_{u} g_{\varepsilon} \vec{T}_{\varepsilon}+g_{\varepsilon}^{2} k_{\varepsilon} \vec{N}_{\varepsilon}$ and $\partial_{t} \partial_{u} x_{\varepsilon}=\partial_{t}\left(g_{\varepsilon} T_{\varepsilon}\right)=$ $\partial_{t} g_{\varepsilon} \vec{T}_{\varepsilon}+g_{\varepsilon} \partial_{t} \nu_{\varepsilon} \vec{N}_{\varepsilon}$ and parts (2) and (3).

Now we are in a position to state the main result of this paper.

THEOREM 5.6. Suppose that $\beta(k, \nu)=\gamma(\nu)|k|^{m-1} k$, where $0<m \leq 2$ and $\gamma$ satisfies (5.1). Let $\Gamma^{0}$ be a smooth regular plane curve such that $\left(k^{0}, \nu^{0}, g^{0}\right)^{T} \in$ $\mathcal{O}_{1} \subset E_{1}$. If $1<m \leq 2$, we also suppose that $\Gamma^{0}$ satisfies the condition (5.6). If the tangential velocity $\alpha^{\varepsilon}$ obeys the conditions (5.4) and (5.5), then there exists $T>0$ and a family of regular plane curves $\Gamma^{t}=\operatorname{Image}(x(., t)), t \in[0, T], x: \overline{Q_{T}}=$ $[0,1] \times[0, T] \rightarrow \mathbb{R}^{2}$ such that

(1) $x, \partial_{u} x \in\left(C\left(\overline{Q_{T}}\right)\right)^{2}, \partial_{u}^{2} x, \partial_{t} x, \partial_{u} \partial_{t} x \in\left(L^{\infty}\left(Q_{T}\right)\right)^{2}$;

(2) $\partial_{t} x \cdot \vec{N}=\beta(k, \nu)$ for any $t \in[0, T]$ and a.e. $u \in[0,1]$, where $k, \nu$, and $\vec{N}$ are the curvatures, the tangent angle, and the unit normal vector of the curve $\Gamma^{t}$.

Proof. It follows from Lemma 5.5, part (4), and the Ascoli-Arzelà theorem that there exists a subsequence of $\left(x_{\varepsilon}\right)$ converging uniformly, i.e., $x_{\varepsilon} \rightarrow x$ in $\left(C\left(\overline{Q_{T}}\right)\right)^{2}$ as $\varepsilon \rightarrow 0^{+}$. By part (5) we also have $\partial_{u} x_{\varepsilon} \rightarrow \partial_{u} x$ in $\left(C\left(\overline{Q_{T}}\right)\right)^{2}$ and $\partial_{t} x, \partial_{u} \partial_{t} x, \partial_{u}^{2} x \in$ $\left(L^{\infty}\left(Q_{T}\right)\right)^{2}$. Again, by (4) and (5) we furthermore have $\nu_{\varepsilon} \rightrightarrows \nu, g_{\varepsilon} \rightrightarrows g$ in $C\left(\overline{Q_{T}}\right)$ and $g>0$. Hence $\vec{T}_{\varepsilon}=g_{\varepsilon} \partial_{u} x_{\varepsilon} \rightrightarrows g \partial_{u} x=\vec{T}$ and $\vec{N}_{\varepsilon} \rightrightarrows \vec{N}$, where $\vec{T}$ and $\vec{N}$ are the unit tangent and normal vectors to the curve $\Gamma^{t}=\operatorname{Image}(x(., t)), t \in[0, T]$. Moreover, $\arg (\vec{T})=\nu$.

Let $t \in[0, T]$ be a fixed time instant. By (1) we have $\left|\partial_{u} \beta^{\varepsilon}\right| \leq M$ and, as a consequence, one has $\beta^{\varepsilon} \rightrightarrows \tilde{\beta}$ in $C\left(S^{1}\right)$. Denote by $b^{\varepsilon}: \mathbb{R} \rightarrow \mathbb{R}$ the inverse function to the increasing function $k \mapsto \beta^{\varepsilon}(k, \nu) / \gamma(\nu), \varepsilon \in[0,1], \beta^{0}=\beta$. Notice that the term $\beta^{\varepsilon}(k, \nu) / \gamma(\nu)$ does not depend on $\nu$. With regard to the regularization assumptions made on $\beta^{\varepsilon}$ we have $b^{\varepsilon} \rightarrow b^{0}=b$ locally uniformly in $\mathbb{R}$. Then for the curvature $k_{\varepsilon}=$ $b^{\varepsilon}\left(\beta^{\varepsilon}\left(k_{\varepsilon}, \nu_{\varepsilon}\right) / \gamma\left(\nu_{\varepsilon}\right)\right)=b^{\varepsilon}\left(\beta^{\varepsilon} / \gamma\left(\nu_{\varepsilon}\right)\right)$ we have the convergence $k_{\varepsilon} \rightrightarrows k=b(\tilde{\beta}) / \gamma(\nu)$ in $C\left(S^{1}\right)$. Thus $\beta(k, \nu)=\tilde{\beta}$. As $\left(\partial_{u}^{2} x_{\varepsilon}\right)$ is bounded in $\left(L^{\infty}\left(S^{1}\right)\right)^{2}$ we have $\partial_{u}^{2} x_{\varepsilon} * \partial_{u}^{2} x$ weak star in $\left(L^{\infty}\left(S^{1}\right)\right)^{2}$. On the other hand, $k_{\varepsilon}=g_{\varepsilon}^{-3}\left(\partial_{u} x_{\varepsilon} \wedge \partial_{u}^{2} x_{\varepsilon}\right) \rightrightarrows k, g_{\varepsilon}^{-3} \rightrightarrows$ $g^{-3}, \partial_{u} x_{\varepsilon} \rightrightarrows \partial_{u} x$. Thus $k(u, t)$ is the curvature of the curve $\Gamma^{t}$ at the point $x=x(u, t)$ for every $t \in[0, T]$ and a.e. $u \in[0,1]$. Finally, as $\left(\partial_{t} x_{\varepsilon}\right)$ is bounded in $\left(L^{\infty}\left(S^{1}\right)\right)^{2}$ we have $\partial_{t} x_{\varepsilon} \stackrel{*}{\rightarrow} \partial_{t} x$ weak star in $\left(L^{\infty}\left(S^{1}\right)\right)^{2}$. Therefore, $\beta^{\varepsilon}=\partial_{t} x_{\varepsilon} \cdot \vec{N}_{\varepsilon} * \partial_{t} x \cdot \vec{N}$ 
as $\varepsilon \rightarrow 0^{+}$. Since $\beta^{\varepsilon} \rightrightarrows \tilde{\beta}=\beta(k, \nu)$ in $C\left(S^{1}\right)$ we conclude $\partial_{t} x \cdot \vec{N}=\beta(k, \nu)$, as claimed.

6. Tangential velocity preserving the relative local length. As was already mentioned in section 2 , the presence of a nontrivial tangential velocity term $\alpha \vec{T}$ in the governing equation (2.4) can prevent the numerically computed solution of (2.2) from forming numerical singularities like, e.g., collapsing of grid points or formation of the so-called swallow tails. The goal of this section is to propose a suitable choice of the functional $\alpha=\alpha(k, \nu, g)$ in such a way that a numerical scheme based on this choice of $\alpha$ will be capable of uniform redistribution of grid points along the computed curve. The main idea behind the construction of $\alpha$ is to analyze the relative local length function defined as the ratio $\frac{\left|\partial_{u} x(u, t)\right|}{L^{t}}$, where $L^{t}$ is the total length of $\Gamma^{t}$ and $\left|\partial_{u} x(u, t)\right|$ represents the local length of $\Gamma^{t}$. The idea is to keep this ratio constant with respect to time, i.e., preservation of the relative local length:

$$
\frac{d}{d t}\left(\frac{\left|\partial_{u} x(u, t)\right|}{L^{t}}\right)=0
$$

for any $u \in[0,1]$ and $t \in I=(0, T)$. Taking into account (3.4) and (3.5) one sees that (6.1) is fulfilled iff

$$
\frac{\partial \alpha}{\partial s}=k \beta(k, \nu)-\frac{1}{L} \int_{\Gamma} k \beta(k, \nu) d s,
$$

where $\Gamma=\Gamma^{t}, L=L(\Gamma), k$ is the curvature of $\Gamma$, and $\beta$ is the given normal velocity function.

In what follows, we will show that there exist geometric quantities $\theta_{1}, \theta_{2}$ such that the tangential velocity function $\alpha$ given by $\alpha=\frac{1}{\theta_{1}} \frac{\partial}{\partial s}\left(\frac{1}{\theta_{2}}\right)$ (see (2.5)) obeys (6.2). We will furthermore prove some a priori estimates for $\alpha$ and $\theta_{i}, i=1,2$, considered as nonlocal operators from the Banach space $E_{1 / 2}$ (see (4.3)) into $C^{2+\sigma}\left(S^{1}\right)$. First we need the following simple lemma.

LEMMA 6.1. Let $\beta^{\varepsilon}$ be a regularization of $\beta$ satisfying regularization assumptions (i)-(iv) from section 5 . Let $\Gamma=$ Image $(x)$ be a $C^{2}$ smooth regular plane curve. Then there exists a unique weak solution $\vartheta \in C^{1}\left(S^{1}\right), \vartheta(0)=\vartheta(1)=0$, of the equation

$$
-\frac{\partial}{\partial s}\left(\frac{\beta^{\varepsilon}(k, \nu)}{k} \frac{\partial \vartheta}{\partial s}\right)=k \beta^{\varepsilon}(k, \nu)-\frac{1}{L} \int_{\Gamma} k \beta^{\varepsilon}(k, \nu) d s .
$$

Furthermore, there exists a constant $C_{4}=C_{4}\left(M_{1}\right)>0$ such that

$$
\max _{\Gamma}|\vartheta| \leq C_{4} \int_{\Gamma} \frac{k}{\beta^{\varepsilon}(k, \nu)} d s \quad \text { and } \quad\left|\partial_{s} \vartheta\right| \leq C_{4} L(\Gamma) \frac{k}{\beta^{\varepsilon}(k, \nu)}
$$

for any $|k| \leq M_{1}$.

Proof. Denote $a=\frac{k}{\beta^{\varepsilon}}, g=\left|\partial_{u} x\right|$, and $f=\frac{1}{L} \int_{\Gamma} k \beta^{\varepsilon}(k, \nu) d s-k \beta^{\varepsilon}(k, \nu)$. Then $0<a<\infty, g>0$, and $a, g, f \in C\left(S^{1}\right)$. Hence

$$
\partial_{u} \vartheta(u)=a(u) g(u)\left(A+\int_{0}^{u} f(v) g(v) d v\right)
$$

for some constant $A$. With regard to the condition $\vartheta(0)=\vartheta(1)=0$ we obtain the existence of a unique weak solution $\vartheta \in C^{1}\left(S^{1}\right)$ and

$$
\vartheta(u)=A \int_{0}^{u} a(\xi) g(\xi) d \xi+\int_{0}^{u} a(\xi) g(\xi) \int_{0}^{\xi} f(v) g(v) d v d \xi,
$$


where $A=-\left(\int_{0}^{1} a(\xi) g(\xi) \int_{0}^{\xi} f(v) g(v) d v d \xi\right)\left(\int_{0}^{1} a(\xi) g(\xi) d \xi\right)^{-1}$. Since $a g \geq 0$ we have $|A|+\left|\int_{0}^{u} f g\right| \leq 2 \max _{\xi} \int_{0}^{\xi}|f| g \leq 2 \int_{0}^{1}|f| g=2 \int_{\Gamma}|f| d s \leq 4 \int_{\Gamma} k \beta^{\varepsilon}(k, \nu) d s \leq$ $4 L(\Gamma) M_{1} C_{2}\left(1+M_{1}^{m}\right)=C_{4} L(\Gamma)$. This, together with (6.4), yields the pointwise estimate for $\left|\partial_{s} \vartheta\right|=\left|g^{-1} \partial_{u} \vartheta\right|$. The bound for $\max |\vartheta|$ now easily follows from the boundary condition $\vartheta(0)=0$.

LEMMA 6.2. Let $\Gamma=\operatorname{Image}(x)$ be a smooth regular plane curve such that $\Phi=$ $(k, \nu, g)^{T} \in \mathcal{O}_{1 / 2} \subset E_{1 / 2},|k| \leq M_{1}$. Let $\beta^{\varepsilon}$ be any regularization of $\beta$ satisfying the regularization assumptions (i)-(iv) from section 5 . Then there exist geometric quantities $\theta_{i}^{\varepsilon}>0, \theta_{i}^{\varepsilon}: \mathcal{O}_{1 / 2} \rightarrow C^{1}\left(S^{1}\right), i=1,2$, such that

$$
\theta_{1}^{\varepsilon} \theta_{2}^{\varepsilon}=\frac{k}{\beta^{\varepsilon}(k, \nu)} \quad \text { and } \quad \alpha^{\varepsilon}=\frac{1}{\theta_{1}^{\varepsilon}} \frac{\partial}{\partial s}\left(\frac{1}{\theta_{2}^{\varepsilon}}\right), \quad \theta_{2}^{\varepsilon}(0)=\theta_{2}^{\varepsilon}(1)=1,
$$

where $\alpha^{\varepsilon} \in C^{1}\left(\mathcal{O}_{1 / 2}, C^{2+\sigma}\left(S^{1}\right)\right)$ is the tangential velocity preserving the relative local length satisfying (6.2). Moreover,

$$
\begin{gathered}
\max _{\Gamma}\left|\theta_{2}^{\varepsilon}(\Phi)\right|+\max _{\Gamma}\left|\theta_{2}^{\varepsilon}(\Phi)^{-1}\right| \leq \exp \left(M_{6} \int_{\Gamma} \frac{k}{\beta^{\varepsilon}} d s\right), \\
\left|\partial_{s} \alpha^{\varepsilon}(\Phi)\right| \leq M_{7}, \quad\left\|\alpha^{\varepsilon}(\Phi)\right\|_{C^{2}} \leq M_{7}\left(1+\left\|\beta^{\varepsilon}(k, \nu)\right\|_{C^{1}}+\|g\|_{C^{1}}\right) ;
\end{gathered}
$$

i.e., $\alpha^{\varepsilon}$ satisfies the hypotheses (5.4) and (5.5).

Proof. Let $\vartheta$ be a solution of (6.3). Define $\theta_{2}^{\varepsilon}=\exp (\vartheta)$ and $\theta_{1}^{\varepsilon}=k /\left(\beta^{\varepsilon} \theta_{2}^{\varepsilon}\right)$. The maximum bounds for $\theta_{2}^{\varepsilon}$ and $\left(\theta_{2}^{\varepsilon}\right)^{-1}$ follow from Lemma 6.1. With regard to Lemma 6.1 we obtain that

$$
\alpha^{\varepsilon}=\frac{1}{\theta_{1}^{\varepsilon}} \frac{\partial}{\partial s}\left(\frac{1}{\theta_{2}^{\varepsilon}}\right)=-\frac{\beta^{\varepsilon}}{k} \frac{\partial}{\partial s} \ln \theta_{2}^{\varepsilon}=-\frac{\beta^{\varepsilon}}{k} \frac{\partial \vartheta}{\partial s}
$$

is a solution of (6.2). Since $\beta^{\varepsilon}$ satisfies the regularization assumption we have $\alpha^{\varepsilon} \in$ $C^{1}\left(\mathcal{O}_{1 / 2}, C^{2+\sigma}\left(S^{1}\right)\right)$. Notice that the estimate for the $C^{2+\sigma}$ norm of $\alpha^{\varepsilon}$ may depend on $0<\varepsilon \leq 1$. It furthermore follows from Lemma 6.1 that $\left\|\alpha^{\varepsilon}(\Phi)\right\|_{C^{0}} \leq M_{6} L(\Gamma)$. With regard to (6.2) we have $\partial_{u} \alpha^{\varepsilon}=\left(k \beta^{\varepsilon}-\right.$ const $) g$, where const $=\frac{1}{L} \int_{\Gamma} k \beta^{\varepsilon} d s$ is a constant. Hence $\left|\partial_{s} \alpha^{\varepsilon}\right|=g^{-1}\left|\partial_{u} \alpha^{\varepsilon}\right| \leq 2 \max _{\Gamma}\left|k \beta^{\varepsilon}(k, \nu)\right| \leq M_{7}$. Furthermore, as $\left\|k \beta^{\varepsilon}(k, \nu)\right\|_{C^{1}} \leq M\left\|\beta^{\varepsilon}(k, \nu)\right\|_{C^{1}}$ and $\mid$ const $\left|\leq \max _{\Gamma}\right| k \beta^{\varepsilon}(k, \nu) \mid$, we have $\left|\partial_{u}^{2} \alpha^{\varepsilon}\right| \leq$ $\mid$ const ||$\partial_{u} g|+| \partial_{u}\left(g k \beta^{\varepsilon}(k, \nu)\right) \mid \leq M_{7}\left(1+\left\|\beta^{\varepsilon}(k, \nu)\right\|_{C^{1}}+\|g\|_{C^{1}}\right)$, and the bound for $\left\|\alpha^{\varepsilon}\right\|_{C^{2}}$ follows. This is why $\alpha^{\varepsilon}$ satisfies the assumptions (5.4) and (5.5).

TheOREM 6.3. Suppose that $\beta(k, \nu)=\gamma(\nu)|k|^{m-1} k$, where $0<m \leq 2$ and $\gamma$ satisfies (5.1). Let $\Gamma^{0}=$ Image $\left(x^{0}\right)$ be a smooth regular plane curve as in Theorem 5.6. Then there exists $T>0$ and a family of regular plane curves $\Gamma^{t}=\operatorname{Image}(x(., t)), t \in$ $[0, T]$ such that

(1) $x, \partial_{u} x \in\left(C\left(\overline{Q_{T}}\right)\right)^{2}, \partial_{u}^{2} x, \partial_{t} x, \partial_{u} \partial_{t} x \in\left(L^{\infty}\left(Q_{T}\right)\right)^{2}$;

(2) the flow $\Gamma^{t}=\operatorname{Image}(x(., t)), t \in[0, T]$ of regular plane curves satisfies the geometric equation

$$
\partial_{t} x=\beta \vec{N}+\alpha \vec{T}
$$

where $\beta=\beta(k, \nu)$ and $\alpha$ is the tangential velocity preserving the relative local length, i.e., $\alpha$ satisfies (6.2) and

$$
\frac{\left|\partial_{u} x(u, t)\right|}{L^{t}}=\frac{\left|\partial_{u} x^{0}(u)\right|}{L^{0}}
$$

for any $t \in[0, T]$ and $u \in[0,1]$. 
Proof. Let us consider the tangential velocity function $\alpha^{\varepsilon}, 0<\varepsilon \leq 1$, satisfying $\partial_{s} \alpha^{\varepsilon}=k_{\varepsilon} \beta^{\varepsilon}\left(k_{\varepsilon}, \nu_{\varepsilon}\right)-\frac{1}{L_{\varepsilon}} \int_{\Gamma_{\varepsilon}} k_{\varepsilon} \beta^{\varepsilon} d s$ whose existence has been verified in Lemma 6.2. Moreover, $\alpha^{\varepsilon}$ is a $C^{1}$ mapping from $\mathcal{O}_{1 / 2} \subset E_{1 / 2}$ into $c^{2+\sigma}\left(S^{1}\right)$ and $\alpha^{\varepsilon}$ satisfies the structural conditions (5.4) and (5.5). By Theorem 5.6 there exists a family of regular plane curves $\Gamma^{t}=\operatorname{Image}(x(., t))$ with the properties as in part (1). To prove (2), we put $\alpha=\partial_{t} x \cdot \vec{T}$ and recall that $g_{\varepsilon}=\left|\partial_{u} x_{\varepsilon}\right| \rightrightarrows g=\left|\partial_{u} x\right|$ as $\varepsilon \rightarrow 0^{+}$. Therefore, $L_{\varepsilon}^{t}=$ $\int_{0}^{1}\left|\partial_{u} x_{\varepsilon}(u, t)\right| d u \rightarrow L^{t}=\int_{0}^{1}\left|\partial_{u} x(u, t)\right| d u$ as $\varepsilon \rightarrow 0^{+}$. Thus $\frac{\left|\partial_{u} x_{\varepsilon}(u, t)\right|}{L_{\varepsilon}^{t}} \rightarrow \frac{\left|\partial_{u} x(u, t)\right|}{L^{t}}$ as $\varepsilon \rightarrow 0^{+}$. On the other hand, since $\alpha^{\varepsilon}$ is the tangential velocity preserving the relative local length we have $\frac{d}{d t} \frac{\left|\partial_{u} x_{\varepsilon}(u, t)\right|}{L_{\varepsilon}^{t}}=0$. Hence $\frac{\left|\partial_{u} x^{0}(u)\right|}{L^{0}}=\frac{\left|\partial_{u} x(u, t)\right|}{L^{t}}$. Therefore, $\alpha$ is the tangential velocity preserving the relative local length and from (6.1), (3.4), and (3.6), we may conclude that $\alpha$ satisfies (6.2).

7. Numerical scheme. In this section we describe a numerical procedure that can be used for computing the curve evolution satisfying the geometric equation (1.1). To this end, we will propose a scheme solving the coupled system of intrinsic heat equation (2.2) for the position vector $x$ and (6.2) for the tangential velocity $\alpha$. A smooth solution $x$ is approximated by discrete plane points $x_{i}^{j}, i=1, \ldots, n, j=$ $0, \ldots, m$, where index $i$ represents space discretization and index $j$ a discrete time stepping. The approximation of a curve in time $j \tau$ (with uniform time step $\tau=\frac{T}{m}$ ) is given by a polygon with vertices $x_{i}^{j}, i=1, \ldots, n$. In order to obtain such an approximation of an evolving curve in the $j$ th time step, we use the following fully discrete semi-implicit scheme:

$$
\frac{1}{2}\left(g_{i}^{j-1}+g_{i+1}^{j-1}\right) \frac{x_{i}^{j}-x_{i}^{j-1}}{\tau}=\frac{x_{i+1}^{j}-x_{i}^{j}}{h_{i+1}^{j-1}}-\frac{x_{i}^{j}-x_{i-1}^{j}}{h_{i}^{j-1}},
$$

$i=1, \ldots, n$, for every $j=1, \ldots, m$. The coefficients in (7.1) (for simplicity we omit upper index $j-1$ ) are given by the following expressions:

$$
\begin{aligned}
g_{i} & =\left|r_{i}\right| \theta_{1, i}, \quad h_{i}=\left|r_{i}\right| \theta_{2, i}, \quad r_{i}=x_{i}-x_{i-1}, \quad \theta_{1, i}=\frac{k_{i}}{\beta_{i} \theta_{2, i}}, \\
k_{i} & =\frac{1}{2\left|r_{i}\right|} \operatorname{sgn}\left(r_{i-1} \wedge r_{i+1}\right) \arccos \left(\frac{r_{i+1} \cdot r_{i-1}}{\left|r_{i+1}\right|\left|r_{i-1}\right|}\right), \\
\nu_{i} & =\arccos \left(r_{i_{1}} /\left|r_{i}\right|\right) \text { if } r_{i_{2}} \geq 0, \quad \nu_{i}=2 \pi-\arccos \left(r_{i_{1}} /\left|r_{i}\right|\right) \text { if } r_{i_{2}}<0, \\
\beta_{i} & =\beta^{\varepsilon}\left(k_{i}, \nu_{i}\right), \quad \theta_{2, i}=\exp \left(\vartheta_{i}\right),
\end{aligned}
$$

and the system (7.1) is subject to the periodic boundary conditions $x_{i+n}^{j}=x_{i}^{j}(i=$ $0,1)$. In order to compute $\vartheta_{i}, i=1, \ldots, n$, governing tangential redistribution of flowing points, we solve

$$
\begin{gathered}
-\frac{\frac{\beta_{i}}{k_{i}}+\frac{\beta_{i+1}}{k_{i+1}}}{\left|r_{i}\right|+\left|r_{i+1}\right|}\left(\vartheta_{i+1}-\vartheta_{i}\right)+\frac{\frac{\beta_{i}}{k_{i}}+\frac{\beta_{i-1}}{k_{i-1}}}{\left|r_{i}\right|+\left|r_{i-1}\right|}\left(\vartheta_{i}-\vartheta_{i-1}\right) \\
=\left|r_{i}\right|\left(k_{i} \beta_{i}-\left(\sum_{l=1}^{n}\left|r_{l}\right| k_{l} \beta_{l}\right)\left(\sum_{l=1}^{n}\left|r_{l}\right|\right)^{-1}\right)
\end{gathered}
$$

for $i=1, \ldots, n$, accompanied by the periodic boundary conditions $\vartheta_{i+n}=\vartheta_{i}(i=$ $0,1)$. 
The system (7.3) can be represented by a symmetric positive semidefinite tridiagonal matrix with kernel containing $n$-dimensional vector each component of which is equal 1 . Since $\sum_{i=1}^{n} b_{i}=0$, where $b_{i}$ are the components of the right-hand side of (7.3), we have assured the existence of a solution that is also unique up to an additive constant. We choose the unique solution by imposing the constraint condition $\vartheta_{0}=\vartheta_{n}=0$.

Then, the linear systems (7.1) can be represented by two symmetric positive definite tridiagonal matrices for which we have the existence and uniqueness of a solution. In each discrete computational time step $j \tau$ the scheme (7.1)-(7.3) leads to solving three tridiagonal systems, namely, one for the redistribution of points along the curve and two for finding the new curve position.

Remark 7.1. The approximation (7.1) can be considered as a full time-space discretization analogy to the backward Euler time semidiscretization scheme

$$
\frac{x^{j}-x^{j-1}}{\tau}=\frac{1}{\theta_{1}^{j-1}} \frac{\partial}{\partial s^{j-1}}\left(\frac{1}{\theta_{2}^{j-1}} \frac{x^{j}}{\partial s^{j-1}}\right), \quad j=1,2, \ldots, m,
$$

of (2.2), where the terms $\theta_{1}, \theta_{2}$ as well as arclength parameterization $s$ are taken from the previous time step $x^{j-1}$, and $\Gamma^{j}=\operatorname{Image}\left(x^{j}\right)$ is considered as a smooth approximation of the evolution in discrete time $j \tau$. Denoting $\delta_{\tau}\left(x^{j}\right)=\left(x^{j}-x^{j-1}\right) / \tau$ and $d s^{j-1}=\left|\partial_{u} x^{j-1}\right| d u, \theta_{1}^{j-1} \theta_{2}^{j-1}=k^{j-1} / \beta^{\varepsilon}\left(k^{j-1}, \nu^{j-1}\right)$, we easily obtain

$$
\delta_{\tau}\left(x^{j}\right)=\tilde{\beta} \vec{N}^{j}+\tilde{\alpha} \vec{T}^{j},
$$

where

$$
\tilde{\beta}=\frac{\left|\partial_{u} x^{j}\right|^{2} k^{j}}{\left|\partial_{u} x^{j-1}\right|^{2} k^{j-1}} \beta^{\varepsilon}\left(k^{j-1}, \nu^{j-1}\right), \quad \tilde{\alpha}=\frac{1}{\theta_{1}^{j-1}} \frac{\partial}{\partial s^{j-1}}\left(\frac{1}{\theta_{2}^{j-1}} \frac{\left|\partial_{u} x^{j}\right|}{\left|\partial_{u} x^{j-1}\right|}\right) .
$$

In the next proposition we show that the backward Euler time discretization scheme generates a discrete curve shortening sequence of plane curves. This result can be considered just as an indication and not a rigorous proof that the sequence of numerically computed discrete polygonal curves is stable in the sense that their length decreases during evolution. The detailed analysis of the stability of the scheme (7.1)-(7.3) is a work in progress and we hope it will be discussed in the forthcoming paper.

Proposition 7.1. Assume $x^{j-1} \in C^{1}\left(S^{1} ; \mathbb{R}^{2}\right), \theta_{1}^{j-1}, \theta_{2}^{j-1} \in C^{1}\left(S^{1} ; \mathbb{R}^{2}\right)$ are such that $\left|\partial_{u} x^{j-1}\right|>0, \theta_{1}^{j-1}>0, \theta_{2}^{j-1}>0$. Then there exists a unique solution $x^{j} \in C^{2}\left(S^{1} ; \mathbb{R}^{2}\right)$ of (7.4). Moreover,

$$
L^{j}+\tau \int_{\Gamma^{j}} \tilde{\beta} k^{j} d s^{j} \leq L^{j-1},
$$

where $L^{j}=\int_{0}^{1}\left|\partial_{u} x^{j}\right| d u$ represents the length of the curve $\Gamma^{j}$. The sequence $\Gamma^{j}=$ Image $\left(x^{j}\right)$ represents a curve shortening discrete flow.

Proof. The existence and uniqueness of a solution $x^{j}$ can be achieved in the same way as was done in [33, Lemma 4.1] in the case $\theta_{1}=\theta_{2}=k / \beta$. To prove the estimate (7.6) we proceed in a similar way as in the continuous case (see (3.5)). Indeed, the time-discrete analogy of the first equation in (3.1) is given by

$$
\delta_{\tau}\left(\partial_{u} x^{j}\right)=\left|\partial_{u} x^{j}\right|\left(\left(\partial_{s^{j}} \tilde{\beta}+\tilde{\alpha} k^{j}\right) \vec{N}^{j}+\left(-\tilde{\beta} k^{j}+\partial_{s^{j}} \tilde{\alpha}\right) \vec{T}^{j}\right)
$$


and therefore

$$
\begin{aligned}
\left|\partial_{u} x^{j}\right| & =\tau \delta_{\tau}\left(\partial_{u} x^{j}\right) \cdot \vec{T}^{j}+\partial_{u} x^{j-1} \cdot \vec{T}^{j}=\tau \delta_{\tau}\left(\partial_{u} x^{j}\right) \cdot \vec{T}^{j}+\left|\partial_{u} x^{j-1}\right| \vec{T}^{j-1} \cdot \vec{T}^{j} \\
& \leq\left|\partial_{u} x^{j-1}\right|+\tau\left(-\tilde{\beta} k^{j}+\partial_{s^{j}} \tilde{\alpha}\right)\left|\partial_{u} x^{j}\right|=\left|\partial_{u} x^{j-1}\right|-\tau \tilde{\beta} k^{j}\left|\partial_{u} x^{j}\right|+\tau \partial_{u} \tilde{\alpha} .
\end{aligned}
$$

Integrating the above inequality over the interval $[0,1]$ yields the bound (7.6). Since $\beta^{\varepsilon} k \geq 0$ we have $\tilde{\beta} k^{j} \geq 0$ and therefore $L^{j} \leq L^{j-1}$; i.e., $\Gamma^{j}$ represents a curveshortening discrete flow.

Remark 7.2. The scheme (7.1)-(7.3) can be derived by using the flowing control volume method (cf. [37]). Let us consider points $x_{i}, i=1, \ldots, n$, belonging to a smooth curve $\Gamma^{t}=\operatorname{Image}(x(., t))$, where $x$ is a solution of (2.2) at time $t$. By $\left[x_{i-1}, x_{i}\right]$ we denote the arc of the curve between the points $x_{i-1}$ and $x_{i}$. Let us consider a control volume $V_{i}$ around $x_{i}$ consisting of part of the arc connecting centers $c_{i}, c_{i+1}$ of $\operatorname{arcs}\left[x_{i-1}, x_{i}\right],\left[x_{i}, x_{i+1}\right]$, respectively. Such a centered control volume is flowing and changing a length during the evolution respecting the new positions of the points $x_{i}$ along the curve. Let us integrate intrinsic diffusion equation (2.2) along the finite volume $V_{i}$. We obtain

$$
\int_{V_{i}} \theta_{1} \frac{\partial x}{\partial t} d s=\left[\frac{1}{\theta_{2}} \frac{\partial x}{\partial s}\right]_{c_{i}}^{c_{i+1}}
$$

Let us consider piecewise linear approximation of $x$, i.e., a polygon connecting points $x_{i}, i=1, \ldots, n$. From (7.2) we can compute constant geometrical quantities $k_{i}, \nu_{i}, \beta_{i}$ for each line segment $\left[x_{i-1}, x_{i}\right]$. The quantity $\vartheta_{i}$ can be computed numerically by solving control volume approximation of the intrinsic equation (6.2). Integrating (6.2) along $\left[x_{i-1}, x_{i}\right]$ (a dual volume to $V_{i}$ ) yields

$$
-\left[\frac{\beta}{k} \frac{\partial \vartheta}{\partial s}\right]_{x_{i-1}}^{x_{i}}=\left|r_{i}\right|\left(k_{i} \beta_{i}-\left(\sum_{l=1}^{n}\left|r_{l}\right| k_{l} \beta_{l}\right)\left(\sum_{l=1}^{n}\left|r_{l}\right|\right)^{-1}\right) .
$$

Approximating $\frac{\partial \vartheta}{\partial s}\left(x_{i}\right)$ by $2 \frac{\vartheta_{i+1}-\vartheta_{i}}{\left(\left|r_{i}\right|+\left|r_{i+1}\right|\right)}$ and $\frac{\beta}{k}\left(x_{i}\right)$ by $\frac{1}{2}\left(\frac{\beta_{i}}{k_{i}}+\frac{\beta_{i+1}}{k_{i+1}}\right)$ we end up with the system (7.3). Now, approximating $\frac{\partial x}{\partial t}$ by $\dot{x}_{i}$ inside $V_{i}$ we obtain from (7.7) the system of ordinary differential equations

$$
\frac{1}{2}\left(\left|r_{i}\right| \theta_{1, i}+\left|r_{i+1}\right| \theta_{1, i+1}\right) \dot{x}_{i}=\frac{1}{\theta_{2, i+1}} \frac{x_{i+1}^{j}-x_{i}^{j}}{\left|r_{i+1}\right|}-\frac{1}{\theta_{2, i}} \frac{x_{i}^{j}-x_{i-1}^{j}}{\left|r_{i}\right|} .
$$

There is a range of possibilities of how to solve this system. In order to obtain the scheme (7.1) we approximate the time derivative by the time difference of the new and previous discrete curve position where all nonlinear terms are taken from the previous time step and linear terms are considered at a new time level. The numerical simulations of section 8 show that such an approximation is sufficient in very general cases regarding accuracy and efficiency of computations. Moreover, using Proposition 7.1 we have guaranteed a kind of stability for numerical computations.

8. Discussion on numerical experiments. In this section we describe numerical results obtained by the algorithm (7.1)-(7.3) for solving the geometric equation (1.1). We test properties of the model and the numerical scheme in evolution of convex as well as nonconvex (and nonrectifiable) initial curves in the presence of nonlinearity and anisotropy in the shape of function $\beta$. The effect of redistribution of discrete 


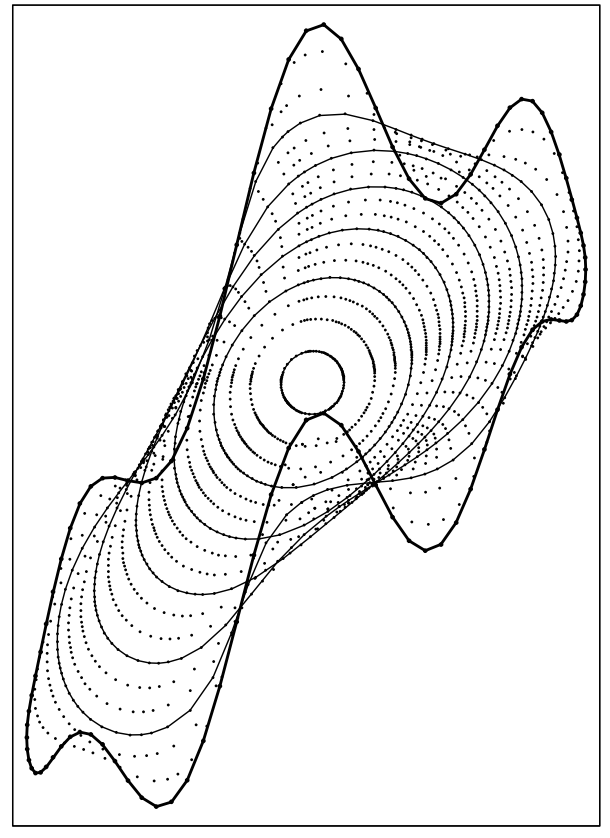

(a)

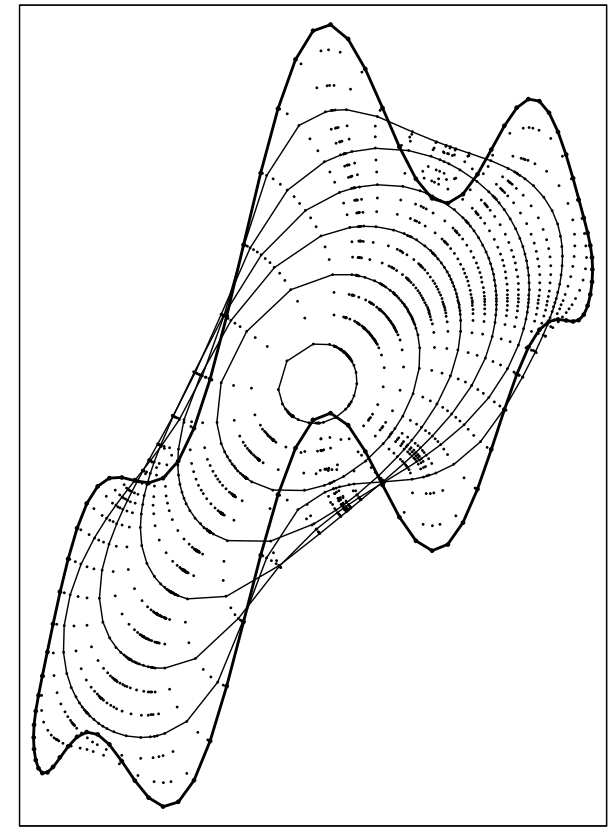

(b)

FIG. 1. $\beta(k)=k$. (a) Discrete evolution using tangential redistribution of points. (b) Without redistribution, only normal component of velocity is used.

points representing an evolving curve is documented in the same time. We have found several examples where the uniform grid redistribution based on relative local length conservation of flowing curve segments is an important tool in correct handling of the curve evolution without other artificial operations like points removing or artificial cutting of the so-called swallow tails. The redistribution of grid points based on (6.2) preserves the initial discretization of a curve and thus makes its discrete representation smooth enough during evolution. First such examples are given in Figures 1(a) and $1(\mathrm{~b})$. In those experiments $\beta(k)=k$; i.e., we have classical curve shortening, and we start with initial curve with large variations in the curvature, namely,

$$
\begin{aligned}
& x_{1}(u)=\cos (2 \pi u), \\
& x_{2}(u)=\frac{1}{2} \sin (2 \pi u)+\sin \left(x_{1}(u)\right)+\sin (2 \pi u)(0.2+\sin (2 \pi u) \sin (6 \pi u) \sin (6 \pi u)),
\end{aligned}
$$

$u \in[0,1]$, and initial discretization is given by uniform division of the range of parameter $u$. The curve is represented by 100 discrete points. Addition of a nontrivial tangential velocity obeying (6.2) leads to the evolution plotted in Figure 1(a). In Figure 1(b) the points move only in the normal direction and one can see their fast merging in several regions and very poor discrete representation in other pieces of the curve. In all experiments we have used the uniform time step $\tau=0.001$. The blowup time for the curvature was $T_{\max }=0.363$. Isoperimetric ratio starting with 3.02 tends to 1.0, which is consistent with Grayson's theorem [24]. In both figures, we plot each 20th discrete time step using discrete points representing the evolving curve, and in each 60 th time step we plot also by piecewise linear curve connecting those points. 


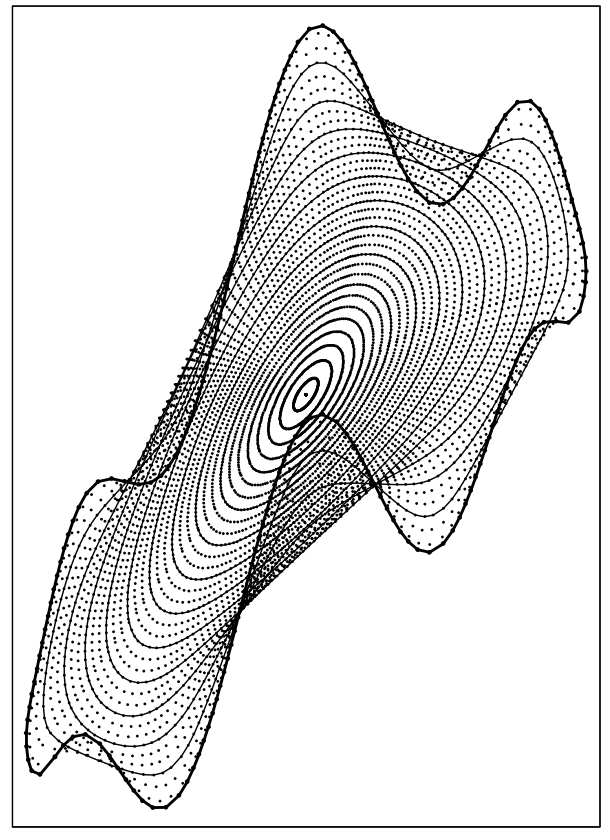

(a)

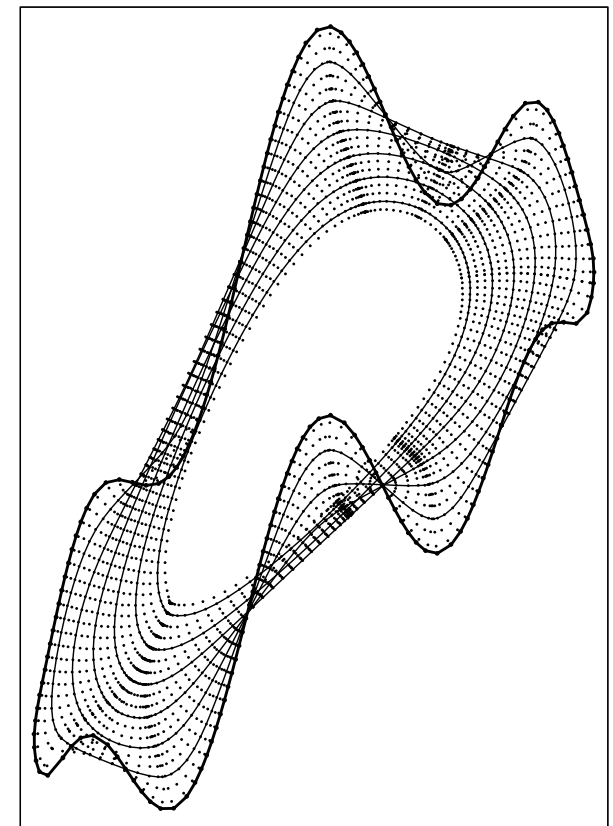

(b)

FIG. 2. $\beta(k)=k^{1 / 3}$. (a) Discrete evolution using tangential redistribution of grids preserving the relative local length. (b) Without redistribution, computation collapses due to vanishing of the local length element $\left|\partial_{u} x\right|$.

In Figures 2(a) and 2(b) we computed affine evolution of the same initial curve for the affine scale $\beta(k)=k^{1 / 3}$. The initial curve has been discretized almost uniformly. In Figure 2(a) we show how this discretization is then preserved in evolution when using the scheme (6.2). The blowup time $T_{\max }=0.694$, a solution converges to an ellipse with the isoperimetric ratio stabilized on 1.33. This is in good agreement with analytical results of Sapiro and Tannenbaum [38]. On the other hand, without any grid redistribution we can see rapid merging of several points leading to degeneracy in the distance $\left|r_{i}\right|$ corresponding to discretization of the term $\left|\partial_{u} x\right|$ and subsequent collapse of computation. In Figure 2(b) one can see evolution until $t=0.38$ just before numerical collapse of a solution.

In the figures below we have shown evolutions of the initial " $\infty$-like" curve. In Figures 3(a) and 3(c) the tangential velocity preserving relative local length has been used, whereas in Figure 3(b) one sees that the computation without tangential redistribution cannot prevent vanishing of the term $\left|\partial_{u} x\right|$. In Figures 4(a) and 4(b) evolutions of general nonconvex curves are plotted.

In Figures 5(a) and 5(b) the affine invariant evolution of initial ellipse with halfaxes ratio $3: 1$ is shown. In Figure 5(a) the exact blowup time $T_{\max }=1.560$, while the numerically computed one is equal to 1.570 using time step $\tau=0.001$ and 100 grid points for curve representation. The half-axes ratio as well as isoperimetric ratio were perfectly conserved during numerical evolution. Without any tangential velocity (i.e., $\alpha=0$ and $\theta_{2}=1$ ), the numerical solution collapses, as should be obvious from Figure 5(b). 


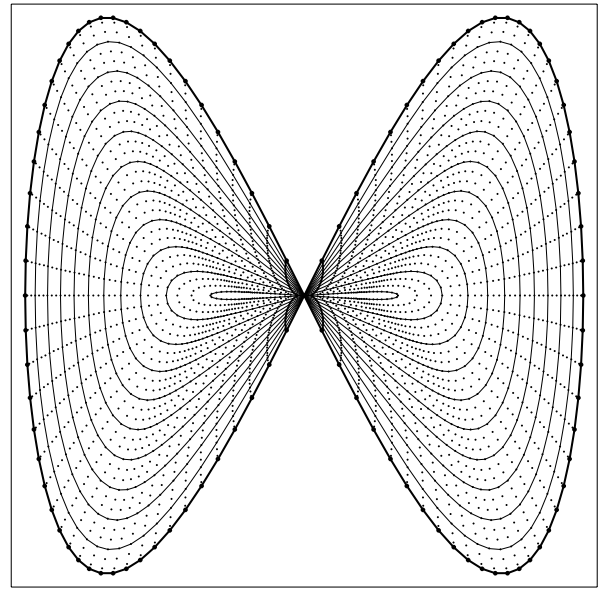

(a)

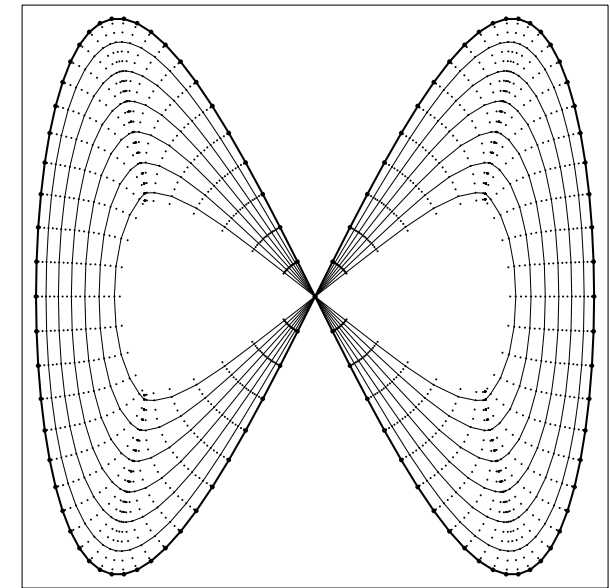

(b)

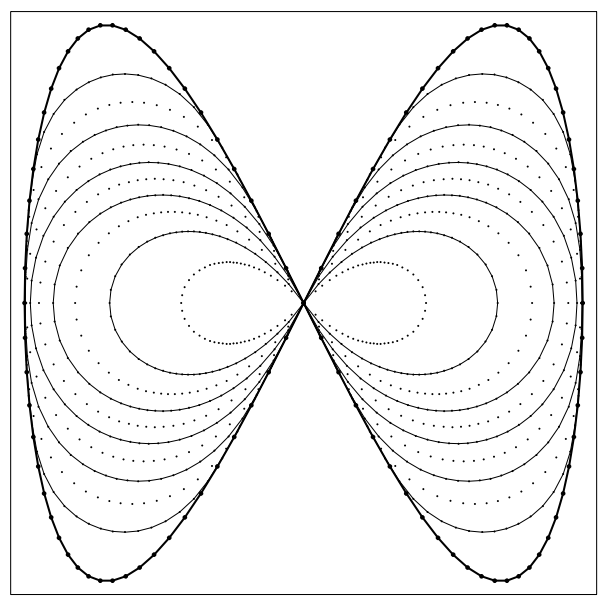

(c)

FIG. 3. $\beta(k)=k^{1 / 4}$. (a) Evolution of " $\infty$-like" curve using redistribution. (b) Evolution of " $\infty$-like" curve without redistribution leading to merging of points. (c) Evolution of " $\infty$-like" curve using tangential redistribution of points.

In Figures 6 and 7 we present various computations including anisotropy in the model. For Figures 6(a)-6(d) we have chosen threefold anisotropy, while for Figures 7(a) and 7(b), a fourfold one. In Figure 6(a) we have computed linear anisotropic evolution of a unit circle by means of (7.1)-(7.3). In Figures 6(b) and 6(c) we have combined anisotropy with a nonlinear function of the curvature. In Figure 6(d) we have chosen the same initial curve and the velocity function as in Figure 6(c), but curves were computed without uniform grid redistribution. Curves are represented by 100 grid points and $\tau=0.001$. In the first numerical experiment shown in Figure 6(a) the numerical blowup time $T_{\max }=0.509$ (the exact one is 0.5 ). In this case the isoperimetric ratio tends to 1.048 and the curve approaches the Wulf shape for such 


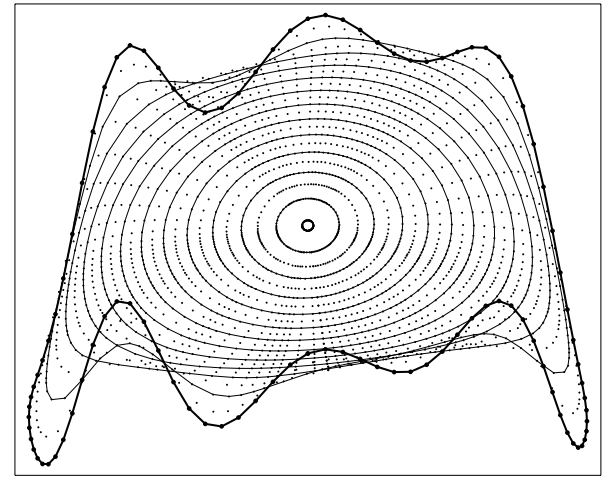

(a)

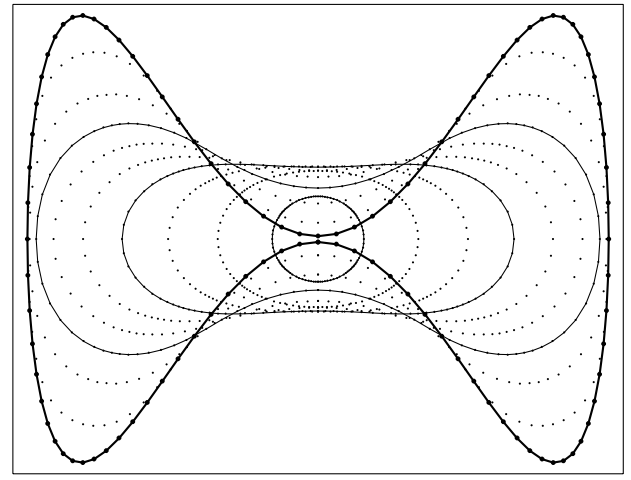

(b)

FIG. 4. $\beta(k)=k^{1 / 2}$. Evolution of general nonconvex curve using tangential redistribution of points.

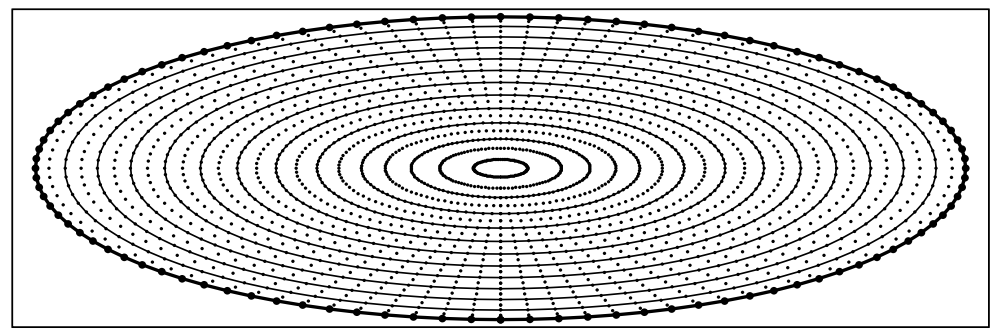

(a)

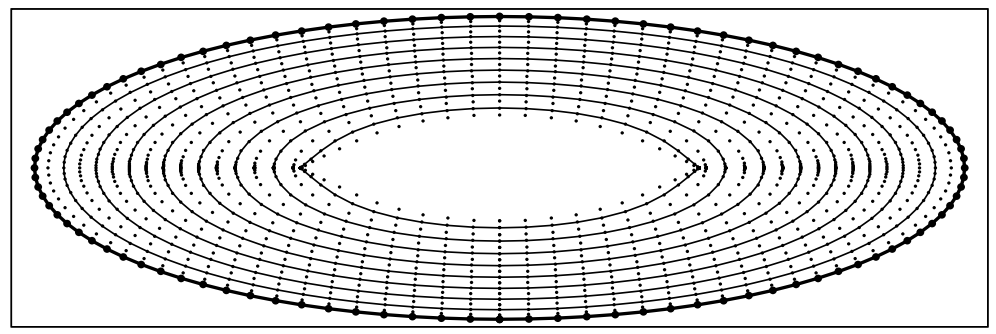

(b)

FIG. 5. (a) Affine invariant motion of ellipse using tangential redistribution of points. (b) Computation using only normal component of velocity.

an anisotropy function. In Figure 6(b) we chose $\beta(k)=k^{m}, m>1$. The evolution is faster, numerical $T_{\max }=0.373(m=2)$, and the asymptotic isoperimetric ratio is 1.014. Taking $\beta(k)=k^{m}, m<1$, the anisotropic evolution is slowed down, numerical $T_{\max }=0.601, m=\frac{1}{2}$, the isoperimetric ratio tends to 1.13 , and the asymptotical shape is more "sharp." In this example one sees that the initial uniform redistribu- 


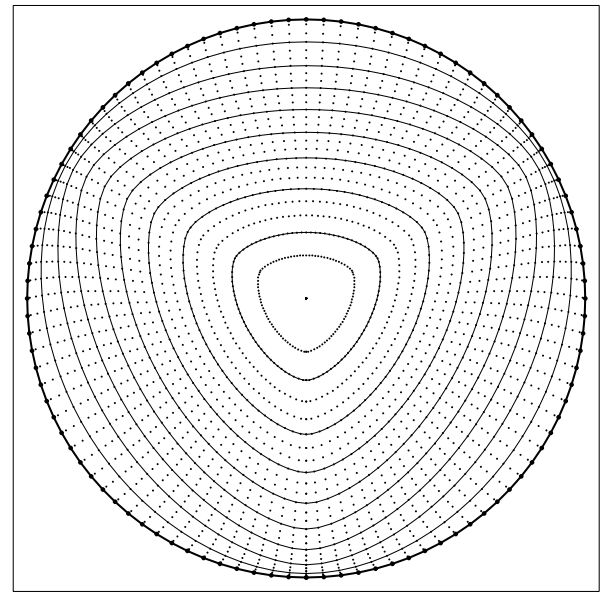

(a)

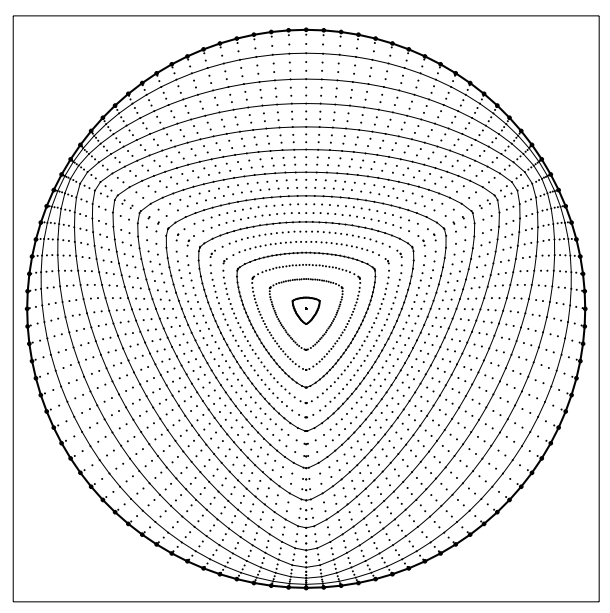

(c)

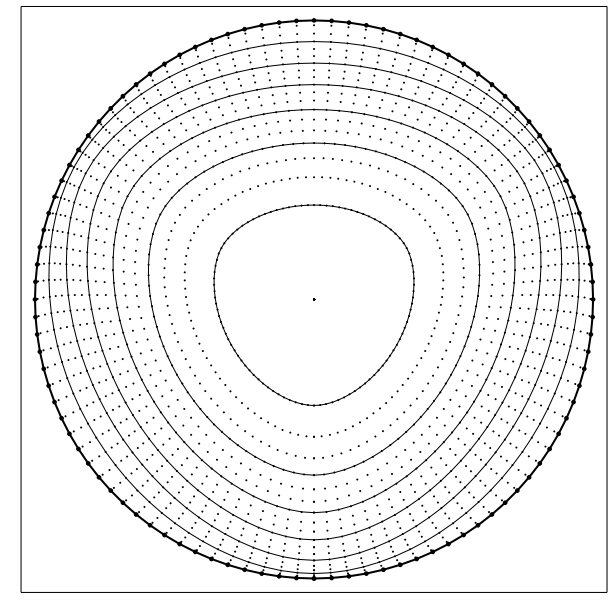

(b)

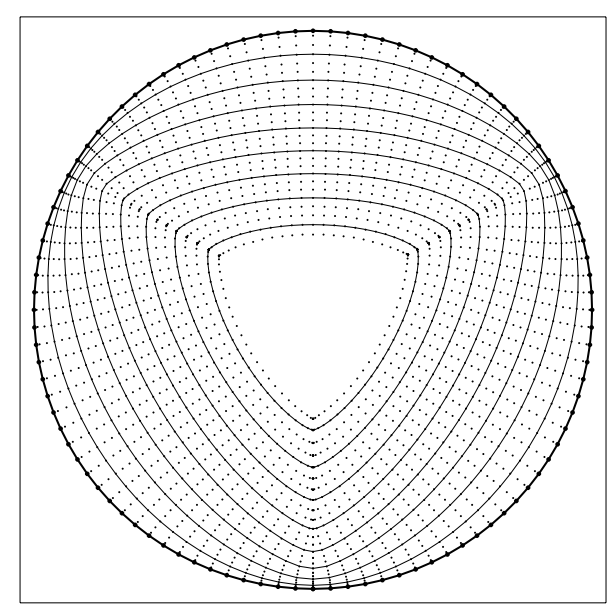

(d)

FIG. 6. $\beta(k, \nu)=(1-7 / 9 \cos (3 \nu)) k$. (a)-(c) Using redistribution. (d) Without redistribution.

tion of grid points is not kept perfectly (in spite of results in Figure 6(b)). It very likely is caused by lack of well-conditioning of linear systems. This phenomenon is an objective of our future study. Further anisotropic experiments are presented in Figures $7(\mathrm{a})-7(\mathrm{c})$, where convergence to "oval square" is observed in both convex and nonconvex cases. The evolution of a nonconvex curve from Figure $7(\mathrm{c})$ is computed also for the case of the threefold anisotropy. Results are plotted in Figure $7(\mathrm{~d})$. The last numerical experiment represents affine invariant evolution of a spiral. In Figure 8 we present several time moments of the motion until it is shrinking to a point.

9. Concluding remarks. In this paper we have studied the generalized mean curvature flow of planar curves. The normal velocity $v$ of the flow is assumed to be a power-like function of the curvature $k$, and it may also depend on a spatial anisotropy 


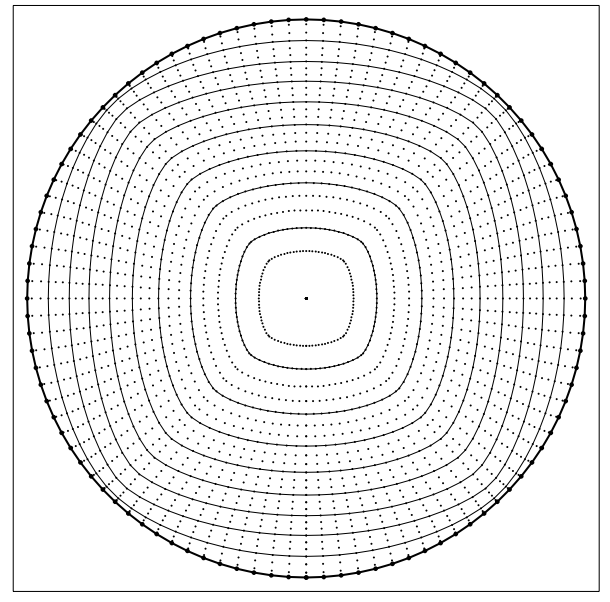

(a)

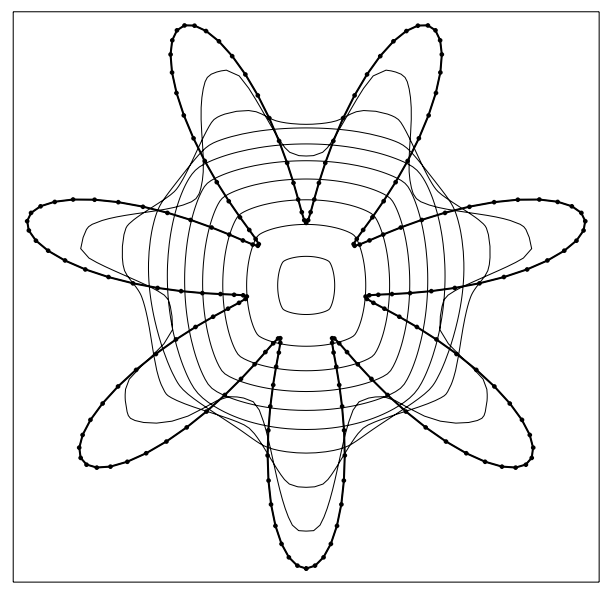

(c)

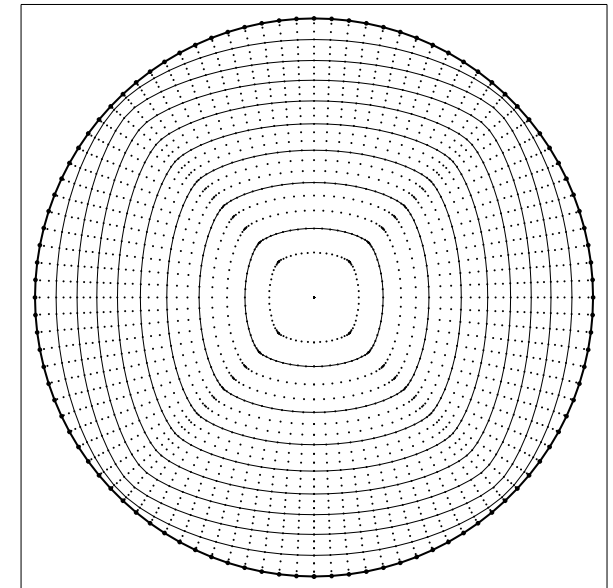

(b)

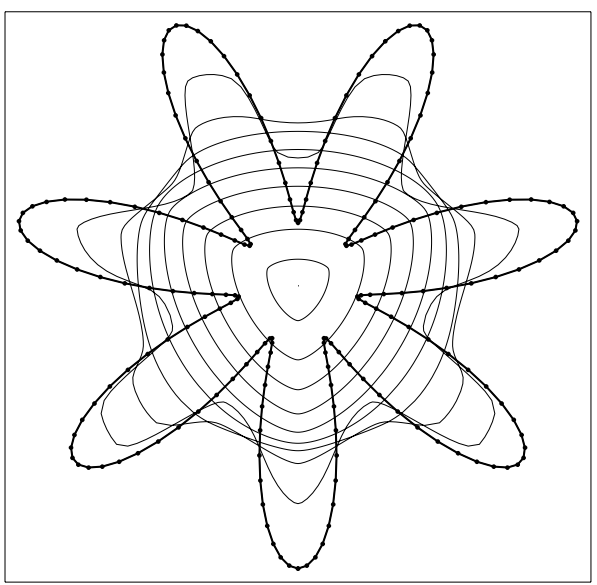

(d)

FIG. 7. $\beta=(1-0.8 \cos (4(\nu-\pi / 4))) k$. (a), (c), (d) Using redistribution. (b) Without redistribution.

$\gamma$, i.e., $v=\gamma k^{m}$, where $m>0$. Our analysis covers both singular $(0<m<1)$ and degenerate $(1<m \leq 2)$ cases. We followed the so-called direct approach. We have proposed and analyzed a governing intrinsic heat equation which is a parabolic equation for the position vector. This model is capable of describing both normal and tangential velocities of an evolving family of plane curves. We have also found that respect to choices of the tangential velocity numerical simulations may exhibit various instabilities. We overcome this difficulty by constructing a suitable tangential velocity functional yielding uniform redistribution of numerically computed grid points.

Acknowledgments. The authors are thankful to the anonymous referees for their valuable comments and for bringing to our attention the recent paper by Andrews. 

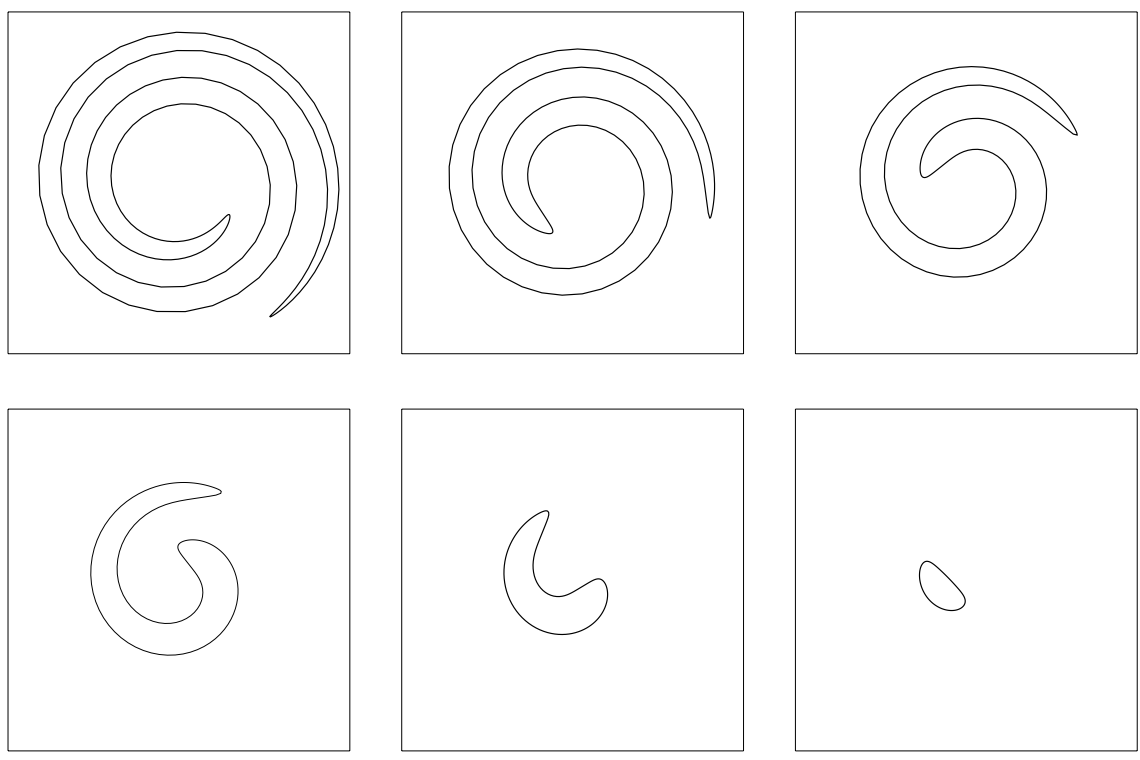

FIG. 8. The sequence of evolving spirals for $\beta(k, \nu)=k^{1 / 3}$ using redistribution. The limiting curve is an ellipse rounded point.

\section{REFERENCES}

[1] U. Abresch AND J. LANGER, The normalized curve shortening flow and homothetic solutions, J. Differential Geom., 23 (1986), pp. 175-196.

[2] L. Alvarez, F. Guichard, P. L. Lions, and J. M. Morel, Axioms and fundamental equations of image processing, Arch. Rational Mech. Anal., 123 (1993), pp. 200-257.

[3] L. Alvarez and J. M. Morel, Formalization and computational aspects of image analysis, Acta Numer., Cambridge University Press, Cambridge, UK, 1994, pp. 1-59.

[4] B. Andrews, Evolving convex curves, Calc. Var. Partial Differential Equations, 7 (1998), pp. $315-371$.

[5] S. B. Angenent, On the formation of singularities in the curve shortening flow, J. Differential Geom., 33 (1991), pp. 601-633.

[6] S. B. Angenent, Parabolic equations for curves on surfaces I: Curves with p-integrable curvature, Ann. Math., 132 (1990), pp. 451-483.

[7] S. B. Angenent, Parabolic equations for curves on surfaces II: Intersections, blow-up and generalized solutions, Ann. Math., 133 (1991), pp. 171-215.

[8] S. B. Angenent, Nonlinear analytic semiflows, Proc. Roy. Soc. Edinburgh Sect. A, 115 (1990), pp. $91-107$,

[9] S. B. Angenent And M. E. GuRTin, Multiphase thermomechanics with an interfacial structure 2. Evolution of an isothermal interface, Arch. Rational Mech. Anal., 108 (1989), pp. 323391.

[10] S. B. Angenent and M. E. Gurtin, Anisotropic motion of a phase interface. Well-posedness of the initial value problem and qualitative properties of the interface, J. Reine Angew. Math., 446 (1994), pp. 1-47.

[11] S. B. Angenent And M. E. GuRTin, General contact angle conditions with and without kinetics, Quart. Appl. Math., 54 (1996), pp. 557-569.

[12] S. B. Angenent, G. Sapiro, and A. Tannenbaum, On affine heat equation for non-convex curves, J. Amer. Math. Soc., 11 (1998), pp. 601-634.

[13] M. Beneš And K. Mikula, Simulations of anisotropic motion by mean curvature-comparison of phase field and sharp interface approaches, Acta Math. Univ. Comenian. (N.S.), 67 (1998), pp. 17-42.

[14] G. Caginalp, The dynamics of a conserved phase field system: Stefan-like, Hele-Shaw, and Cahn-Hilliard models as asymptotic limits, IMA J. Appl. Math., 44 (1990), pp. 77-94.

[15] V. Caselles, R. Kimmel, G. Sapiro, and C. Sbert, Minimal surfaces based object segmentation, IEEE Trans. Pattern Anal. Mach. Intell., 19 (1997), pp. 394-398. 
[16] K. Deckelnick, Weak solutions of the curve shortening flow, Calc. Var. Partial Differential Equations, 5 (1997), pp. 489-510.

[17] G. DzIUK, Algorithm for evolutionary surfaces, Numer. Math., 58 (1991), pp. 603-611.

[18] G. Dziuk, Convergence of a semi discrete scheme for the curve shortening flow, Math. Models Methods Appl. Sci., 4 (1994), pp. 589-606.

[19] G. DzIUK, Discrete anisotropic curve shortening flow, SIAM J. Numer. Anal., 36 (1999), pp. 1808-1830.

[20] C. M. Elliott, M. Paolini, and R. Schätzle, Interface estimates for the fully anisotropic Allen-Cahn equation and anisotropic mean curvature flow, Math. Models Methods Appl. Sci., 6 (1996), pp. 1103-1118.

[21] M. Gage And R. S. Hamilton, The heat equation shrinking convex plane curves, J. Differential Geom., 23 (1986), pp. 285-314.

[22] P. M. GIRÃO, Convergence of a crystalline algorithm for the motion of a simple closed convex curve by weighted curvature, SIAM J. Numer. Anal., 32 (1995), pp. 886-899.

[23] P. M. GiRÃo AND R. V. KoHn, Convergence of a crystalline algorithm for the heat equation in one dimension and for the motion of a graph by weighted curvature, Numer. Math., 67 (1994), pp. 41-70.

[24] M. GraYson, The heat equation shrinks embedded plane curves to round points, J. Differential Geom., 26 (1987), pp. 285-314.

[25] M. GuRTin, Thermomechanics of Evolving Phase Boundaries in the Plane, Clarendon Press, Oxford, UK, 1993.

[26] A. Handlovičová, K. Mikula, and A. Sarti, Numerical solution of parabolic equations related to level set formulation of mean curvature flow, Comput. Visualization Sci., 1 (1998), pp. 179-182.

[27] M. Kass, A. Witkin, And D. Terzopoulos, Constraints on deformable models: Recovering 3D shape and nongrid motion, Artificial Intelligence, 36 (1988), pp. 91-123.

[28] M. Kimura, Accurate numerical scheme for the flow by curvature Appl. Math. Letters, 7 (1994), pp. 69-73.

[29] M. KImURA, Numerical analysis for moving boundary problems using the boundary tracking method, Japan J. Indust. Appl. Math., 14 (1997), pp. 373-398.

[30] R. Malladi, J. Sethian, And B. Vemuri, Shape modeling with front propagation: a level set approach, IEEE Trans. Pattern Anal. Mach. Intell., 17 (1995), pp. 158-174.

[31] K. Mikula AND J. KaČUR, Evolution of convex plane curves describing anisotropic motions of phase interfaces, SIAM J. Sci. Comput., 17 (1996), pp. 1302-1327.

[32] K. Mikula, Solution of nonlinear curvature driven evolution of plane convex curves, Appl. Numer. Math., 21 (1997) pp. 1-14.

[33] K. Mikula AND D. ŠEvČOvič, Solution of nonlinearly curvature driven evolution of plane curves, Appl. Numer. Math., 31 (1999), pp. 191-207.

[34] L. MoIssan, Affine plane curve evolution: A fully consistent scheme, IEEE Trans. Image Process., 7 (1998), pp. 411-420.

[35] R. Nochetto, M. PaOlini, And C. Verdi, Sharp error analysis for curvature dependent evolving fronts, Math. Models Methods Appl. Sci., 3 (1993), pp. 711-723.

[36] S. Osher And J. Sethian, Fronts propagating with curvature dependent speed: Algorithm based on Hamilton-Jacobi formulation, J. Comput. Phys., 79 (1988), pp. 12-49.

[37] S. Patankar, Numerical Heat Transfer and Fluid Flow, Hemisphere, New York, 1980.

[38] G. Sapiro and A. Tannenbaum, On affine plane curve evolution, J. Funct. Anal., 119 (1994), pp. $79-120$.

[39] A. Schmidt, Computation of three dimensional dendrites with finite elements, J. Comput. Phys., 125 (1996), pp. 293-312.

[40] A. Schmidt, Approximation of crystalline dendrite growth in two space dimensions, Acta Math. Univ. Comenian. (N.S.), 67 (1998), pp. 57-68.

[41] J. Sethian, Numerical algorithm for propagating interfaces: Hamilton-Jacobi equations and conservation laws, J. Differential. Geom., 31 (1990), pp. 131-161.

[42] J. Sethian, Level Set Methods. Evolving Interfaces in Geometry, Fluid Mechanics, Computer Vision, and Material Science, Cambridge University Press, Cambridge, UK, 1996.

[43] J. Sethian, Adaptive fast marching and level set methods for propagating interfaces, Acta Math. Univ. Comenian. (N.S.), 67 (1998), pp. 3-16.

[44] T. UshiJima And S. YAZAKI, Convergence of a crystalline algorithm for the motion of a closed convex curve by a power of curvature $V=K^{\alpha}$, SIAM J. Numer. Anal., 37 (2000), pp. $500-522$. 